\title{
Trivium
}

Revue franco-allemande de sciences humaines et sociales - Deutsch-französische Zeitschrift für Geistesund Sozialwissenschaften

$13 \mid 2013$

Entre morale, politique et religion : la cohésion sociale selon Emile Durkheim

\section{Emile Durkheim: Das Soziale als Gegenstand der Wissenschaft. Vom Moralischen zum Politischen?}

\author{
Jean-Claude Chamboredon
}

Traducteur : Michael Bischoff

\section{OpenEdition}

\section{Journals}

Édition électronique

URL : http://journals.openedition.org/trivium/4452

DOI : $10.4000 /$ trivium.4452

ISSN : 1963-1820

Éditeur

Les éditions de la Maison des sciences de l'Homme

Référence électronique

Jean-Claude Chamboredon, «Emile Durkheim: Das Soziale als Gegenstand der Wissenschaft Vom Moralischen zum Politischen? », Trivium [Online], 13 | 2013, online erschienen am 28 Februar 2013, abgerufen am 10 Dezember 2020. URL : http://journals.openedition.org/trivium/4452 ; DOI : https:// doi.org/10.4000/trivium.4452

Ce document a été généré automatiquement le 10 décembre 2020.



Les contenus des la revue Trivium sont mis à disposition selon les termes de la Licence Creative Commons Attribution - Pas d'Utilisation Commerciale - Pas de Modification 4.0 International. 


\title{
Emile Durkheim: Das Soziale als Gegenstand der Wissenschaft. Vom Moralischen zum Politischen?
}

\author{
Jean-Claude Chamboredon
}

Traduction : Michael Bischoff

\section{NOTE DE L'ÉDITEUR}

Wir danken Herrn Jean-Claude Chamboredon für die freundliche Genehmigung, diesen Artikel in deutscher Übersetzung zu publizieren. Es sei darauf hingewiesen, dass der Anmerkungsapparat gegenüber dem Originaltext stark gekürzt wurde.

Nous remercions M. Jean-Claude Chamboredon de nous avoir accordé l'autorisation de traduire ce texte pour le présent numéro. Nous signalons que la majeure partie des notes de bas de page de l'article original a été supprimée.

1 Durkheim ist zu einem Streitobjekt in den Auseinandersetzungen zwischen den Sozialwissenschaften bzw. zwischen verschiedenen Denktraditionen innerhalb einzelner Sozialwissenschaften, insbesondere der Soziologie, geworden. Die Aneignung dieses Emblems (um den Ausdruck zu benutzen, den er selbst zur Analyse des Totemismus verwendete) erfordert eine genealogische Rekonstruktion und die Durchsetzung einer Interpretation des Werkes. Daher die Wandlungen Durkheims, die sich in der Bestimmung der Bedeutung seines Werkes und dem Gewicht der verschiedenen Teile abzeichnen. Vor allem in Frankreich entbrannte dieser Wettkampf um das Werk in den 1960er Jahren, als die Soziologie in einer Phase universitärer Expansion und Institutionalisierung durch eine Rückwendung zu den Gründern des Fachs nach Sicherheit, Ansehen und wissenschaftlichen Modellen suchte. Diese Entwicklung folgte übergangslos auf das Ende der akademischen Fortführung des Durkheimismus und die hagiographische Pflege des Gedächtnisses dieses Mannes und seiner Schule. ${ }^{1}$ Da Durkheim im angelsächsischen Raum auch schon früher zum Gegenstand der Analyse und der Forschung gemacht wurde ${ }^{2}$, kam es zu merkwürdigen 
Begegnungen und Interferenzen, von denen einige bei Gelegenheit noch zur Sprache kommen werden. Dabei sind am wenigsten merkwürdig durchaus nicht jene Fälle, in denen ein mit diversen Originalteilen in Amerika »zusammengebauter« Durkheim in einem Hin und Her, das an manche Tricks des Import-Export-Geschäfts erinnert, den Franzosen zurückverkauft wurde, die davon gar nichts bemerkten (Durkheim wird oft auf dem Weg über die Kommentare von Merton und Parsons gelesen). Angesichts des gegenwärtigen Bedeutungsrückgangs und der intellektuellen Krise des Fachs, von der die theoretische Zersplitterung und der Zerfall in diverse Kirchen (wenn nicht gar Sekten) zeugen, dient die Rückkehr zu Durkheim als Rückversicherung oder Zuflucht. Wenn die Beschäftigung mit den Ursprüngen nicht der Vorbereitung einer kritischen Wiederaufnahme und heuristischen Anwendung der theoretischen Konstruktionen der Begründer des Fachs dient, kann sie nur eine fetischistische Regression oder eine scholastische Kanonisierung darstellen.

Der Kampf um die Aneignung und die genealogischen Bemühungen (zu irgendetwas ist jedes Unglück gut) haben zu einer Vervielfachung der Arbeiten geführt (Neuausgaben und Textsammlungen, Sozialgeschichte und Soziologie der Durkheimschule ${ }^{3}$, interpretierende Aufsätze ${ }^{4}$ ), die es ermöglichen, die Werke genauer kennenzulernen, die Bedeutung des wissenschaftlichen Unternehmens besser einzuschätzen und $\mathrm{zu}$ erkennen, was darin zum Gegenstand einer Neuinterpretation gemacht werden könnte. Aber das gilt nur, wenn es gelingt, Durkheims Zersplitterung in zahlreiche fetischisierte Facetten zu überwinden, deren jede Gegenstand eines gesonderten Kults in den verschiedenen Soziologenpopulationen bildet; nur wenn es außerdem zu verhindern gelingt, dass die genealogische Rekonstruktion sich zum Ursprungsroman entwickelt; nur wenn es schließlich auch gelingt, das sakralisierende Verhältnis zu den Texten zu vermeiden, das darin besteht, zeitbedingte theoretische Positionen, partielle Theoretisierungen (zu deren Entstehungsbedingungen nach P. Feyerabend ein gewisser Fluss und eine gewisse semantische Offenheit gehören) in eine geschlossene allgemeine Theorie zu verwandeln, die allenfalls noch einer Kommentierung und Systematisierung bedarf.

Insbesondere kann man unter den verschiedenen, in den Kommentaren und historischen Darstellungen vermittelten Durkheimbilder verschiedene Durkheims unterscheiden: Einen »Methodologen« Durkheim (der in Der Selbstmord Methoden zur Analyse der Beziehungen zwischen verschiedenen Variablen, mulitivariate Analyseverfahren, Methoden der ökologischen Analyse und dergleichen anwendete und sie in Regeln der soziologischen Methode systematisierte), jenen "Methodologen«, der nach einer Reise nach Amerika, insbesondere an die Columbia University, nach Frankreich zurückkehrte. ${ }^{5}$ Einen Durkheim der allgemeinen Theorie (den grand theorist, um einen Begriff aufzugreifen, den C. Wright Mills auf andere anwandte); entweder vor allem seit den Analysen von T. Parsons ${ }^{6}$ - als Schöpfer der Theorie der sozialen Ordnung im Sinne internalisierter normativer Anforderungen und Vorläufer des Strukturfunktionalismus oder als Denker der modernen Gesellschaft (Arbeitsteilung und Differenzierung der von organischer Solidarität geprägten Gesellschaft), der zugleich deren Krise (Anomie) theoretisch erfasste - dies vor allem in den Analysen von R. Nisbet.7 Einen Durkheim als Oberhaupt einer Schule und Begründer einer Wissenschaft, als Organisator eines Gemeinschaftsunternehmens (l'Année sociologique), eines gelungenen Versuchs, ein neues Fachgebiet zu institutionalisieren und an der Universität zu etablieren. Das von J. Ben-Davids Wissenschaftssoziologie inspirierte 
Buch von Terry Clark $^{8}$ ist der systematischste Versuch, diesen Aspekt des Durkheimismus $\mathrm{zu}$ isolieren und zu konstruieren, aber auch zahlreiche Arbeiten aus dem Bereich der historischen Soziologie, die sich mit der Durkheimschule befassen, teilen diese Tendenz. ${ }^{9}$ Angesichts all dieser positiven Bilder sollte man vielleicht auch dem teilweise aus P. Nizans Die Wachhunde übernommenen Bild Durkheims als eines antimarxistischen Ideologen einen Platz einräumen, das D. Lindenberg in seinen Studien über die Einführung des Marxismus in Frankreich zeichnet. ${ }^{10}$

Diesen verschiedenen Facetten muss man schließlich noch die eines epistemologischen Durkheim hinzufügen oder überlagern, in der jene Aspekte seines Werkes in den Vordergrund rücken, die eine Theorie der Erkenntnis des Sozialen statt einer speziellen Gesellschaftstheorie vorschlagen, außerdem die wissenschaftliche Polemik gegen die Illusionen des Artifizialismus und der Transparenz sowie der wissenschaftliche Anspruch (die siegesgewissen und grundlegenden Zielsetzungen, die sich unter der damals verehrten, heute verabscheuten Maske des Positivismus verbergen). Die Situation des Anfangs hat in der Tat gewisse Vorzüge, denn sie zwingt zur grundlegenden Explikation bestimmter Voraussetzungen, und das gegen die Interpretationsmonopole, die andere Fachgebiete für sich beanspruchen, oder gegen die Evidenzillusion des Common sense. Das heißt auch, dass die Rückkehr zum Augenblick der Gründung (»Durkheim revisited«) nicht nur die Funktion einer Wallfahrt zu den Ursprüngen hat (das Modell der Geschichte des Fachgebiets als Andacht und Stärkung im Sinne einer säkularisierten Form von Theologie), sondern dass sie die Möglichkeit eröffnet, die zu Anfang getroffenen Entscheidungen, auf denen das Fachgebiet aufbaut, zu erkennen (um sie - wie im Taufversprechen - zu erneuern oder um sie in Frage zu stellen): den Bruch mit wissenschaftlichen Traditionen oder mit vorbegrifflichem Wissen, aber möglicherweise auch mit Nachlässigkeiten, mit Ignoranz und mit vorurteilsbedingten Ausschlüssen.

Diese verschiedenen Figuren (die man systematisch rekonstruieren könnte, indem man eine Logik der Rollen in der wissenschaftlichen Gemeinschaft mit einer Logik der Positionen im epistemologischen Raum kombinierte) bedeuten keine Vorentscheidung hinsichtlich dessen, was aus den verschiedenen Aspekten des Durkheimschen Werkes (Theorie der Arbeitsteilung, Anomie, Integration, Religion usw.) in den theoretischen Umarbeitungen wird, die sich aus den Systematisierungsbemühungen und/oder der empirischen Anwendung ergeben. Die Wiederaufnahme und Neuinterpretation der theoretischen Themen und die Umgestaltung des sozialen Bildes des Soziologen Durkheim haben die aus der philosophischen Tradition Frankreichs hervorgegangenen Durkheimbilder ausgelöscht, die einen Fortbestand der intellektuellen Konstellation der 1930er Jahre markierten; man denke hier an den Positivismus, die Laienscholastik (Thibaudet), die Moraltheorie und das Dogma des kollektiven Bewusstseins (Brunschvicg), deren letzte Nachklänge man in Raymond Arons Analysen zu Durkheim ${ }^{11}$ findet.

6 Die beiden Bücher von J.-C. Filloux und B. Lacroix ${ }^{12}$ tragen dazu bei, das Bild Durkheims als Theoretiker $\mathrm{zu}$ kultivieren, und vernachlässigen vielleicht allzu sehr das des Methodologen und vor allem das des Schuloberhaupts (abstrakte Werkanalyse der Entwicklung der Theorie im Kollektivwerk, Entwicklungen, die manche Aspekte zu erhellen und in ihre Bestandteile zu zerlegen vermögen). Es sind die beiden ersten Bücher, die in Frankreich nach den letzten Hervorbringungen der Durkheimschen Tradition (Davy und Cuvillier) und der französischen Rezeption der angelsächsischen 
Arbeiten die neue Art der Kommentierung des Durkheimschen Werks umsetzen. Beide Bücher sind Dissertationen und präsentieren in dem für akademische Produktionen typischen Tempo zwei verschiedene Durkheimbilder. Das Buch von Filloux (eine philosophische Dissertation) zeichnet ein Durkheimbild, das durchdrungen ist von den Themen, die Mitte der 1960er Jahre gerade Konjunktur hatten (Konzentration auf die Konstruktion einer Architektonik des Sozialen in Gestalt einer soziologischen Theorie G. Gurvitch; Frage nach den Determinierungsebenen - marxistische Fragestellung; nach dem Verhältnis zwischen soziologischer Theorie und politischer Praxis). Das Buch von Lacroix (eine juristische Dissertation) zeichnet ein Durkheimbild, das mehr der Theorielastigkeit der 1970er Jahre geschuldet ist (es befasst sich in erster Linie mit dem epistemologischen Bruch, der Konstruktion des Begriffssystems einer Theorie). Gleichfalls gemeinsam ist ihnen die bedeutende Stellung, die der Psychoanalyse in der Interpretation des Werkes und vor allem des wissenschaftlichen Ehrgeizes seines Autors eingeräumt wird. In thematischer Hinsicht schließlich - bei B. Lacroix liegt der Fokus auf der Rekonstruktion einer Theorie des politischen Systems, die sich in Durkheims Werken finden soll, während J.-C. Filloux das Verhältnis der Theorien des Soziologen zu den zeitgenössischen Sozialtheorien und vor allem zum Sozialismus in den Mittelpunkt rückt - gestatten es beide Bücher, die Stellung der politischen Frage in Durkheims Werk zu erhellen. Das Buch von B. Lacroix treibt sogar den Versuch einer genealogischen Rekonstruktion bis an seine Grenzen und macht Durkheim zum Gründungsvater einer von der Soziologie unterschiedenen Politikwissenschaft. Wir wollen die beiden Bücher zum Anlass nehmen, nach Stellung und Inhalt der politischen Frage bei Durkheim zu fragen: Ist die Soziologie eine Antwort auf die politische Krise? Findet sich bei Durkheim der Entwurf einer Politikwissenschaft? In methodischer Hinsicht werden wir zwei Themenbereiche kritisieren, die diese Bücher recht gut illustrieren: die hermeneutischen Schwierigkeiten, die sich aus der Vorstellung eines intellektuellen Projekts ergeben (ursprüngliche Charakterisierung eines schöpferischen Projekts als ausdrückliche und gänzlich ausgebildete Intention; solipsistische Vorstellung des schöpferischen Subjekts); die Deformationen, zu denen die Rekonstruktion des Werkes in der Darstellung als fertige Lehre führt - ein scholastischer Systematisierungseffekt, wenn man so will. Neben diesen speziellen Diskussionen werden wir auch auf das Verhältnis zwischen der politischen Krise (im Umfeld der 1880er Jahre), der in Durkheims Werk (vor allem in Der Selbstmord) thematisierten moralischen Krise und der sozialen Krise sprechen müssen. Gilt es nicht, Durkheims theoretische Unternehmung von der sozialen Frage her zu untersuchen, wobei die einander folgenden Umgestaltungen des Begriffssystems und die thematischen Verschiebungen als ebenso viele Versuche zu werten sind, diese Frage zu verschieben?

\section{Sozialwissenschaft und politische Kunst}

Bernard Lacroix' Unterfangen, Durkheim als Gründervater der Politikwissenschaft darzustellen, stützt sich auf vier Operationen: Betonung der terminologischen Schwankungen (Durkheim benutzte nicht immer und nicht von Anfang an den Ausdruck "Soziologie« zur Kennzeichnung seiner Arbeit); Privilegierung der in der Anfangszeit (1885-1888) entstandenen Texte, die vor allem auf die Lektüre deutscher Ökonomen und Gesellschaftstheoretiker verweisen, um die Bedeutung politischer Fragen für seine Fragestellung zu belegen; Konzentration der Aufmerksamkeit auf die 
Physik der Sitten und des Rechts (Leçons de sociologie) als Entwurf einer politischen Soziologie Durkheims; Aufweis der politischen Krise Frankreichs in den 1870er und 1880er Jahren als Ausgangspunkt der Probleme, die Durkheim in seine wissenschaftliche Fragestellung umsetzt. Die erste Operation ist in weiten Teilen nur ein Streit um Worte. Jenseits dieses Streits berührt sie allerdings auch Probleme der akademischen Strategie und der institutionellen Einbindung des Durkheimschen Unternehmens. Die zweite und die dritte Operation - die Verlagerung der Aufmerksamkeit auf das Korpus der Durkheimschen Texte - werfen die Frage auf, welche Stellung man den frühen Texten zuweisen und wie man die Physik der Sitten und des Rechts interpretieren sollte (als Abhandlungen zur politischen Wissenschaft oder zur Moral). Die vierte Operation gibt Anlass zu der Frage, wie man die "Krise« zu interpretieren hat, auf deren Grundlage sich Durkheims Denken entfaltete: als politische Krise, als soziale Krise oder als Krise der Moral? Aber auch zu der Frage nach dem Zeitraum dieser Krise: die Konstellation von 1870 bis 1880 oder die von 1880 bis 1890 ?

Die Schwankungen in der Bezeichnung des Forschungsfeldes, mit dem Durkheim sich befassen will, zeigen nur geringfügige Variationen: "Sozialwissenschaft « im Singular und im Plural konkurriert leicht mit "Soziologie", das häufiger und systematischer verwendet wird..$^{13}$ Es stimmt allerdings, dass "Soziologie" einerseits unter der Missbilligung durch den akademischen Spiritualismus leiden konnte, der sich in den 1880er Jahren gegen den Comteismus durchsetzte, und andererseits unter sprachpuristisch begründeten Bedenken gegenüber diesem aus einem griechischen und einem lateinischen Bestandteil zusammengesetzten Wort, doch die Ausdrücke "Soziologie« und "Soziologe" werden (eine Zeitlang in Konkurrenz mit "sociologiste«) seit den ersten, 1885 erschienenen Besprechungen regelmäßig verwendet. Die These einer Unbestimmtheit des Vokabulars wirkt daher gezwungen. Jenseits der Frage nach der Bezeichnung des Forschungsgebiets stellt sie die Frage nach der Bedeutung dieser Bezeichnung: In welchen Bereich des Wissens fügt sich der Versuch zur Schaffung einer Soziologie ein? Was wird damit eingeschlossen und was ausgeschlossen? Wie verortet sie sich im Verhältnis zu den institutionalisierten Fachgebieten und Traditionen? Zunächst einmal kann man hier die Ablehnung der vagen, in der Taxonomie der Académie verwendeten Bezeichnung "sciences morales et politiques" herauslesen, weil die damit umschriebenen Fachgebiete einerseits spekulative, weitgehend historisch ausgerichtete Reflexionen umfassen und andererseits von einem liberalen, großen systematischen Konstruktionen feindlich gesinnten Denken beherrscht sind, das im Zweiten Kaiserreich und seit der Julimonarchie die Oberhand gewonnen hat (siehe das später, nämlich 1900 in einer Darstellung der Geschichte der Soziologie in Frankreich vorgetragene, harte Urteil über die Mittelmäßigkeit dieses Denkens, »die intellektuelle Flaute, die der Mitte des Jahrhunderts zur Unehre gereicht «. ${ }^{14}$ Vor allem aber ist darin die Ablehnung von Denktraditionen zu erblicken, die lediglich darauf abzielen, allein auf der Grundlage einer durch historische Beispiele erhellten Reflexion oder des philosophischen Common sense praktische Handlungsanweisungen zu formulieren. Das Ziel ist die Begründung einer Wissenschaft des Sozialen und nicht die Kodifizierung einer Kunst - ein Gegensatz, den Durkheim häufig durch den Vergleich mit dem Verhältnis zwischen Physiologie und Medizin verdeutlicht. ${ }^{15}$ (C. Bernard ist - wie oft vergessen wird, weil es allzu oft wiederholt worden ist; das sind die Folgen gelehrten Papageientums - Durkheims große wissenschaftliche Leitfigur.) Die distanzierte Haltung gegenüber der Strömung der "politischen Wissenschaften« hängt mit dem 
methodologischen Postulat des Determinismus zusammen. Die Fachgebiete, die man äußerstenfalls und fast um den Preis eines Anachronismus unter diesem Ausdruck zusammenfassen kann, haben als Doktrinen des staatlichen Handelns teil an den Illusionen der Willkürlichkeit sozialer Tatsachen, des künstlichen Charakters der Institutionen (vertragsrechtliche Darstellungen des sozialen Bandes), der Führungstätigkeit großer Männer - all das Positionen, gegen die Durkheim unermüdlich polemisiert. Insbesondere verwirft er die in der traditionellen politischen Philosophie verbreitete spekulative Tradition der Reflexion über den politischen Vertrag: Die Vorstellung eines Gesellschaftsvertrags (ob nun Hobbesscher oder Rousseauscher Prägung) nährt die Illusionen hinsichtlich des Artifizialismus des Sozialen und des Atomismus der sozialen Subjekte. So versucht Durkheim am Ende seines Aufsatzes "L'état actuel des études sociologiques en France«, die Vorteile und die Hindernisse der französischen Denktraditionen für die Entwicklung der Soziologie aufzuzeigen. Im Blick auf die im Dienste der Klarheit stehende übermäßige Vereinfachung demonstriert er, dass dieses Denken die Erforschung der Gesellschaft in einer Reflexion über die Beziehungen zwischen Individuen einschließt: „Unter diesen Umständen ginge es bei soziologischen Problemen immer nur um die Frage, wie diese Individuen leben können, sodass sie sich gegenseitig nicht oder möglichst wenig behindern. Das ist in der Tat der Charakter der politischen Philosophie des 18. Jahrhunderts, die bis vor kurzer Zeit auch die unsrige war.« Und er verweist auf die Orte, an denen diese Ideologie überlebt hat: »bei den immer noch zahlreichen und mächtigen orthodoxen Ökonomen und ihren Schülern; unsere juristischen Fakultäten sind deren letztes Asyl ${ }^{16}{ }^{16}$ Das Hauptziel der Durkheimschen Polemik im Bereich der Sozialwissenschaften bildet die Ökonomie, ein weiteres Indiz für das wenig ausgeprägte Profil dessen, was Lacroix durch "Retrojektion" als "Politikwissenschaft» herausarbeitet.

9 Möglicherweise entfernte das methodologische Postulat eines soziologischen Determinismus Durkheim von der Analyse des politischen Systems, die im Verdacht stand, die philosophische Illusion der Allmacht der »Dekrete« von Regierenden oder Gesetzgebern zu nähren. So schreibt er im Blick auf Comtes zentrale Gedanken: »Die Institutionen der Völker konnten nicht länger als das Produkt des mehr oder weniger aufgeklärten Willens von Fürsten, Staatsmännern oder Gesetzgebern angesehen werden, sondern mussten als notwendige Folgen physisch determinierter Ursachen gelten. $\ll^{17}$ Und schließlich ist Durkheims Grundeinstellung hinsichtlich des Verhältnisse der Soziologie zu den praktischen Problemen der Politik und der Organisation der Gesellschaft zu berücksichtigen: Er beansprucht das Recht zum Umweg und hält es sogar für eine Pflicht, die Antworten auf unmittelbare Fragen der Praxis aufzuschieben, und dies mit dem Hinweis auf die Jugend der Wissenschaft, die er zu entwickeln gedenkt: ein Aufschub (eine epoché, wenn man so will), der in seiner Rechtfertigung eigentlich nur als vorläufige Haltung gedacht war, sich dann aber endlos fortsetzt und zur Moral des Wissenschaftlers wird. Allerdings stellt sich die Frage, ob nicht die Politik, die damit explizit und offiziell zurückgestellt wird (also eher Zensur als Ausschluss, eher aufschiebende Vorsicht als methodologische Vorsichtsmaßnahme), hinterrücks wieder ins Werk zurückkehrt. Wir werden darauf zurückkommen.

10 Von den Problemen der Bezeichnung und Benennung geht man allzu schnell über zur institutionellen Einordnung des von Durkheim kultivierten Fachgebiets. Die oben angesprochenen Abwandlungen in der Namengebung verdienten keine so große Aufmerksamkeit, wenn hier kein Zusammenhang mit Problemen der Soziologie und der 
Erkenntnisphilosophie bestünde. Die Bezeichnungen (Selbstbezeichnungen oder Benennungen durch Kommentatoren und Kritiker) des Fachgebiets, das Durkheim und die Durkheimianer zu entwickeln versuchen, werfen die Frage auf, wo auf der Landkarte des Wissens dieses Fachgebiet zu verorten wäre. Hier begegnen wir einem weiteren Irrtum der in der Soziologie (oder der Sozialgeschichte) gängigen genealogischen Methode: Da man rückschreitend vorgeht, projiziert man häufig die gegenwärtigen Aufteilungen auf die Vergangenheit und weist ihr im Sinne einer einfachen Verlängerung, wie man die Fluchtlinien in einer Zeichnung verlängert, die in der Gegenwart anzutreffenden Grenzen und Spaltungslinien $\mathrm{zu}^{18}$ Das gilt in besonderem Maße für die Soziologie, der Soziologen und Historiker einen autonomen Status zubilligen, obwohl sie (und vor allem die Durkheimsche Soziologie) ihren Bestätigungsort und ihren Ursprung in der Philosophie hat. ${ }^{19}$ Diese zu Anachronismen führende Tendenz ist nun mit zwei wichtigen Themen des hagiographischen Diskurses bezüglich der Wissenschaft verbunden, der sich vielfach im Gewande der Soziologe oder Sozialgeschichte der Wissenschaft ausbreitet und wiederholt. Dabei handelt es sich einerseits um die vulgärhegelianische Vorstellung einer Notwendigkeit der aktuellen Aufteilung der Fachgebiete und andererseits um den Gründungsmythos. Darin wird Durkheim (wie anderwärts Freud, Marx oder beliebig andere, je nach dem betrachteten Fachgebiet) als Gründervater einer Wissenschaft gezeichnet, die selbstbewusst mit den Illusionen der Nichtwissenschaft bricht und den Rahmen des neuen Fachgebiets absteckt.

Um Durkheims epistemologische Vorschriften und Polemiken ebenso wie seine akademischen und wissenschaftlichen Strategien angemessen zu erfassen, müssen wir, wie es scheint, mindestens zwei Kontexte unterscheiden, in denen die Soziologie sich verortet und zur Geltung bringt. Auf diese Weise kann man auch die Vielfalt der Strategien und die Labilität der Gegensätze berücksichtigen, die in den auf Fixierung ausgerichteten geschichtlichen Darstellungen außer Acht bleiben. Die Wissenschaftslandkarten, die man rekonstruieren muss, wenn man die Struktur des Labyrinths erkennen will, in dem Durkheim seinen Weg sucht, könnten letztlich mit der akademischen und insbesondere universitären Organisation, dem "Streit der Fakultäten«, wie Kant dies genannt hat, und mit dem Konflikt der Fachgebiete innerhalb der Fakultäten verbunden sein. Zwei Gegensatzstrukturen sind hier zu nennen. Die eine stellt den Wissenschaften die Künste oder den Fakultäten die écoles gegenüber wie Theorie und Praxis, dem zweckfreien das berufsorientierte Wissen. In dieser Struktur stehen die philosophische und die naturwissenschaftliche Fakultät der juristischen und der medizinischen Fakultät (früher écoles) gegenüber, die berufspraktisches Wissen vermitteln und die Studenten nicht zu Wissenschaftlern ausbilden, sondern eben zu Angehörigen bestimmter Berufe. Dieser Mythos, der eine positivistische Form (Kult der Wissenschaft) oder eine idealistische Form (Kult der Theorie und der Spekulation, Wissenschaft als Hüterin der großen Werte) annehmen kann - und dies je nach Fachgebiet oder école (die klassischen humanistischen Fächer berufen sich bevorzugt auf die idealistische Form, die "Wissenschaftler" der experimentellen Disziplinen, etwa die Psychologen, auf die positivistische Variante) dieser Mythos ist symptomatisch (weil eine Verkehrung der realen Situation) für die Machtwünsche der Angehörigen der in der universitären Hierarchie ganz unten angesiedelten Fakultäten, und zwar nach der Zahl der eingeschriebenen Studenten (die philosophische und die naturwissenschaftliche Fakultät - ein Gemeinplatz in der Geschichte der Hochschullehre - haben praktisch keine regulären Studenten und sind 
in erster Linie Prüfungsinstanzen, die Scheine und Diplome ausstellen), nach ihrer Qualität und nach ihrer Ausrichtung auf einzelne Berufe (Lehre). Auf dieser Landkarte steht die an der philosophischen Fakultät gelehrte Soziologie im Gegensatz zu all jenen Fachgebieten, die eine Praxis kodifizieren: das Recht (mit Ausnahme der wissenschaftlichen Formen wie der vergleichenden Rechtsgeschichte), die Ökonomie (die an der juristischen Fakultät gelehrt wird) und auch die Politikwissenschaften (die allerdings auf der universitären Landkarte noch kaum in individualisierter Gestalt vorkommt), die moderne Form der »Kameralwissenschaften«.

Doch es gibt noch eine zweite Art von Gegensatz, der weniger mit der Organisation der Universität zusammenhängt und gänzlich innerhalb der "theoretischen" oder "wissenschaftlichen « Fakultäten (der philosophischen und der naturwissenschaftlichen Fakultäten) bleibt. Diese Gegensatzstruktur ist in ihrer vollendeten Form als ein System von Wissenschaften organisiert, nimmt aber jenseits dieser extremen Ausprägung auch schwächere Formen an. Die erste, in der philosophischen Fakultät zu findende ist jene, in der positive Fachgebiete (die Psychologie, die den Vorteil hat, die positivistische Wende als erste vollzogen $\mathrm{zu}$ haben, dann auch die Soziologie) spekulativen Fachgebieten (der Philosophie) oder literarischen Traditionen (einer gewissen Form des literarischen Humanismus) gegenübergestellt werden. Auf dieser Ebene bewegt sich der Gegensatz zwischen Soziologie und Geschichte (einem Fachgebiet, das mit der sozialen Welt denselben Gegenstand hat wie die Soziologie, aber nicht das Ziel verfolgt, durch typologische Konstruktionen eine Wissenschaft des Allgemeinen zu entwickeln). Die zweite Form dieses Gegensatzes, diesmal nach einem enzyklopädischen Modell konstruiert, ist jene, die das System jener Wissenschaften organisiert, welche Lebewesen zum Gegenstand haben: Biologie, Psychologie, Soziologie - eine Gliederung, die aus der Comteschen Klassifikation der Wissenschaften übernommen worden ist und bei Durkheim unter dem Einfluss des epistemologischen Diskontinuitätsgedankens eines Boutroux gedacht wird. ${ }^{20}$ Der epistemologische Diskurs, die wissenschaftlichen Polemiken und die Strategien Durkheims und der Durkheimianer müssen in diesem Kontext von Gegensätzen gesehen werden, wenn man ihre Logik und ihre Triebkräfte verstehen will. Diese Gegensatzstrukturen folgen einem Gesamtmodell, das man als Einschachtelung bezeichnen könnte (Gegensätze innerhalb der philosophischen Fakultät, zum Beispiel Soziologie/Moral oder Soziologie/Geschichte; sodann Gegensätze zwischen den Fakultäten, zum Beispiel Soziologie/Rechtswissenschaft oder Wissenschaft/praktische Berufe usw.), sofern man nicht vergisst, dass diese Gegensätze - wie man sagen könnte, wenn man hierher überträgt, was Goody im Blick auf die Techniken der Schrift oder der Schaubilder und Tabellen gezeigt hat ${ }^{21}$ - nicht mit der Systematik und Strenge eines kodifizierten theoretischen Systems organisiert sind, sondern als Möglichkeiten, die eine gewisse Flexibilität behalten, wobei die klassifikatorische Unschärfe gerade die Bedingung der Möglichkeit strategischer Spiele (intellektueller und akademischer Strategien) bildet. Man könnte zeigen, dass Durkheims Polemiken und Problemdarstellungen (in programmatischen Texten oder Bilanzen, in Regeln der soziologischen Methode und diversen Aufsätzen, die sein Werk durchziehen und in denen er Schaubilder erstellt und wissenschaftliche Programme nachzeichnet, die den Stand des Fachgebiets erläutern), seine Forschungsstrategien und die Abgrenzung seines Problemfeldes ihren Sinn in den oben beschriebenen Gegensatzstrukturen erhalten. Das dürfte genügen, um die anachronistische und von wissenschaftlicher Hagiographie durchdrungene Illusion eines Wissenschaftsgründers zu zerstreuen, der zwischen einer klar vorgegebenen Alternative zu wählen hätte, 
nämlich zwischen Soziologie und Politikwissenschaft. Doch die Probleme der Abgrenzung des Fachgebiets, der epistemologischen und institutionellen Verortung innerhalb des Wissens, unterscheiden sich von zwei anderen Problemen, die einer systematischen Analyse bedürfen, dem der Gegenstände (der Frage, welche Stellung Durkheims Soziologie den Gegenständen einer Politikwissenschaft zuweist) und dem der Ursprünge (der Frage, welche Bedeutung die politische Krise zu Beginn der Dritten Republik einerseits für die Entstehung der wissenschaftlichen Berufung Durkheims und andererseits innerhalb seiner Fragestellungen besitzt). Genau diese Fragen stellen die Bücher von Filloux und Lacroix, auch wenn sie hinsichtlich der Antwort nicht einer Meinung sind, denn Filloux zeichnet das Bild eines Moralphilosophen Durkheim, dessen Intimfeind der Sozialismus ist, während Lacroix ihn als Gründer einer Wissenschaft darstellt, die letztlich auf politische Theorie abzielt. Zunächst jedoch bedarf es einer Abschweifung in Gestalt einer methodischen Erörterung.

\section{Hermeneutik oder Soziologie der Werke}

Den Studien von J.-C. Filloux und B. Lacroix sind zwei methodische Entscheidungen gemeinsam, deren eine, wie man sagen könnte, von der Faszination für die Hermeneutik des schöpferischen Projekts, deren andere von der philologischen Illusion des Beliebens des Scholiasten zeugt. Die erste Entscheidung besteht darin, das Werk abstrakt zu fassen, den Schöpfer zu isolieren, das Werk als opus, als geschlossenes Bedeutungssystem, und den Autor als Monade zu behandeln, dessen Verhältnis zur Geschichte allein im Sinne des Gegensatzes zwischen einem Ausdrucksprojekt und den solchem Ausdruck entgegenstehenden Hindernissen zu denken. Das hat eine gewisse hermeneutische Willkür zu Folge und die Nichtbeachtung der Konstitutions- und Entwicklungsbedingungen des Projekts. Die Kohärenz des Werkes, die Abfolge seiner Restrukturierungen und sein Verhältnis zu wechselnden Fragestellungen wie auch die ideologischen und theoretischen Konstellationen bleiben unberücksichtigt. Insgesamt fügt sich diese methodische Position ein in eine Arbeitsteilung zwischen Spezialisten der Kommentierung des Werkes, dem Klan der Exegeten, und Spezialisten der Erforschung der Karrieren und der institutionellen Geschichte der Schule, dem Klan der "Soziologen«. Wollte man genauer differenzieren, müsste man bei Durkheims Kommentatoren innerhalb der Klasse der Exegeten noch zwischen den Theoretikern (zu denen Filloux und Lacroix gehören) und den Methodologen unterscheiden (vor allem den Kommentatoren der Methodologie in Der Selbstmord). Diese Methodenwahl ist auf der Ebene der Behandlung der Texte in funktionaler Hinsicht mit einer weiteren Wahlentscheidung verbunden: Unter dem Vorwand literarischen - also sakralen oder rituellen - Respekts vor einem zu kommentierenden Textkorpus führen die Exegeten Willkür und Belieben in die Behandlung des von ihnen untersuchten Werkes ein. Für die Zwecke des Glossierens nimmt diese Willkür zwei scheinbar gegensätzliche, in Wirklichkeit aber eng miteinander verwandte Formen an: Entweder beschließt man, Texte zu isolieren, ohne etwas über ihr Verhältnis zum Rest des Werkes zu sagen (das tut B. Lacroix, der sich auf frühe Schriften von 1883 bis 1887 und die Physik der Sitten und des Rechts konzentriert), oder, wenn die Untersuchung sich auf das Gesamtwerk bezieht, reduziert man es auf eine Ansammlung von Zitaten, ohne dabei die Möglichkeit einer Überprüfung an der Kohärenz des Gesamtwerks - oder zumindest 
eines größeren Teils davon - und an der zeitlichen Reihenfolge zu nutzen (so ist es bei J.-C. Filloux).

Diese zutiefst mit der Tradition der Kommentierung von Werken übereinstimmenden Vorannahmen der Hermeneutik finden sich selbst noch bei den jüngsten Nachfolgern des kritischen Diskurses (das Werk als Totalität, dessen Kohärenz der Kommentar aufzeigen muss, indem er es als System rekonstruiert; der Autor als isolierte Person im Pantheon der Denker, dessen kritischer Diskurs das Bild wiederherstellt; die Theorien als das Wesentliche fassende Gebilde, von Anfang an in ihrer Vollendung gegeben, wie sie in dem Bild der Doktrinen gegeben sind, das die Geschichte des betreffenden Fachgebiets zeichnet). Das von Sartre inspirierte Modell des expressiven Entwurfs verleiht der Darstellung des Schöpfers als Monade neue Kraft. In den unmittelbarer von der Psychoanalyse inspirierten Formen ist es die Idee einer Urkrise, einer Persönlichkeitsstruktur, die ihren Ausdruck im schöpferischen Entwurf des Werkes findet. So ist für Filloux Durkheims prophetischer Entwurf die Auflösung des Widerspruchs zwischen den väterlichen Plänen für Emile Durkheim (Rabbiner) und der Selbstbehauptung (Laisierung der Berufung). Dieses Modell der Entstehung eines Wesens des Werkes und des expressiven Entwurfs wird nur wenig verändert durch zwei Abwandlungen, die eine einfache Peripetie in die Entwicklung des Werkes einführen: das Schema des "Bruchs" und das der "neurotischen Krise«.

Diese »Kammer«-Geschichte (auch wenn es sich um die camera obscura der Psychoanalyse handelt) schließt das Schicksal des Werkes in die individuelle Entwicklung eines Schöpfers ein und beschränkt den Sinn eines Werkes auf seinen unmittelbaren Inhalt (ohne Verhältnisse des Gegensatzes oder der Nähe zu anderen Werken innerhalb eines Problemfeldes zu berücksichtigen, durch die es erst seine Bedeutung erhält). Zur Kritik dieses Vorgehens reicht es indessen nicht aus, wenn man sagt, es reproduziere das Verhältnis des Exegeten und die philologische Illusion (das Werk als System, der Exeget als Schöpfer) und liefere das Werk tatsächlich meist der Willkür von Rekonstruktionen aus, die dem Bedürfnis jüngerer Doktrinen entsprechen: bei J.-C. Filloux etwa die Rekonstruktion eines Durkheimschen Systems, dem Gurvitchs Topik der Stufen des Sozialen (vom stark Kristallisierten zum weniger stark Kristallisierten) übergestülpt wird; oder bei B. Lacroix das Schicksal, das der metaphorischen Bezeichnung des "Substrats" im Versuch der Rekonstruktion einer Durkheimschen politischen Theorie widerfährt. Diese Kommentarentscheidungen bzw. -positionen führen zu einer Verfestigung, Immobilisierung und Systematisierung von veränderlichen und labilen Analogien und Metaphern, deren semantische Unschärfe gerade die Voraussetzung für die theoretische Erfindungsgabe darstellt. ${ }^{22}$ Außerdem verkennt dieses Vorgehen die Soziologie der Ausdrucksbedingungen und der Voraussetzungen für die Entstehung des Werkes und vergisst das Verhältnis zwischen dem Schöpfer und seinem Publikum wie auch seinem sozialen Kontext. Hier fehlt es an einer Analyse der Beziehung des Schöpfers zum Publikum und an einer Soziologie der Ausdrucksbeziehung, die den Autor mit bestimmten Gruppen (oder Quasigruppen) verbindet; es fehlt an einer Analyse des ideologischen Bereichs; an einer zeitlichen Gliederung des Werks und insbesondere seiner Entwicklung im Zusammenhang mit der zeitlichen Gliederung der übergeordneten Felder (des philosophischen Feldes, des sozialen und politischen Feldes), deren Entwicklungen den Problemkontext bestimmen, in dem das Werk sich entwickelt, was die Frage nach dem Ineinander oder 
Nacheinander der Konstellationen aufwirft; es fehlt an einer Analyse des Prozesses, durch den das Werk sich zu einer Tradition verfestigt.

Diese Fragen kann man auch im Blick auf die Wende stellen, zu der es angeblich um 1895 in Durkheims Werk kam. Auf welche empirischen Belege stützt sich die Diagnose solch eines Bruchs? Auf Durkheims eigene Aussagen hinsichtlich der »Entdeckung» der Bedeutung der Religion ${ }^{23}$; auf Anzeichen einer moralischen und psychologischen Krise im Zusammenhang mit einer Wende in seiner beruflichen Laufbahn ${ }^{24}$; auf Hinweise von Mauss (im Vorwort $\mathrm{zu}$ Le Socialisme) auf eine "wissenschaftliche" Wende und die Abkehr von der Beschäftigung mit einer kritischen Analyse des sozialen Denkens und der Erforschung des Sozialismus; eine institutionelle Veränderung: die Gründung der Année sociologique, eines Organs, das eine Schule um sich versammelte, eine stärker kollektive Arbeitsweise erforderte und zum Instrument der Ausarbeitung eines Lehrkorpus wurde (eine Werkstatt der Kritik und des gemeinschaftlichen Schaffens, wenn man so will); und schließlich eine Veränderung im Werk: eine thematische Verschiebung von Über soziale Arbeitsteilung hin zu Der Selbstmord oder genauer noch eine Veränderung der Bedeutung bestimmter Begriffe (vor allem »Anomie«, eines Begriffs, der bei Durkheim mit der Analyse der Krise der modernen Gesellschaften verbunden war). B. Lacroix verweist nur auf die beiden ersten Indizien und auf das letztgenannte (die gewollte Originalität seines Buchs sollte ihnen zur Geltung verhelfen). Auch J.-C- Filloux räumt dem letzten Indiz einen Platz ein. Man braucht jedoch nur die verschiedenen Gründe zusammenzunehmen, damit sich Zweifel an dem bestechenden und Befriedigung versprechenden Modell eines »Bruchs« innerhalb des Werkes einstellen (ein in vitro, im abgeschlossenen Raum des Opus wie auch in der Einzigartigkeit und im Solipsismus des Schöpfungsentwurfs vollzogener Bruch). Die fehlende Synchronisierung dieser Kalender, das Wunder einer Selbstdiagnose an einem Wendepunkt des Werkes (zudem noch im ganz speziellen Kontext einer Polemik formuliert), die Geringfügigkeit mancher Hinweise und schließlich das gänzliche Fehlen jeglicher Verbindung zur akademischen, intellektuellen, politischen und sozialen Geschichte innerhalb der aufgezeigten Veränderungen - all das muss jeden beunruhigen, der nicht gänzlich der Faszination einer symptomalen Lektüre erlegen ist.

Zur Ausdrucksbeziehung zwischen dem Soziologen (oder in anderen Fällen dem Schriftsteller, dem Schaffenden) und den von Gruppen getragenen Fragestellungen wollen wir zwei Hinweise aus dem Bereich der sozialen und politischen Fragen anführen. Danach können wir nach der Position, von der aus Durkheim spricht, und nach der institutionellen Stellung seiner halbprophetischen Aussagen fragen und die ideologische Konstellation (oder Abfolge von Konstellationen) sowie die konkurrierenden Fragestellungen beschreiben, vor deren Hintergrund sein Werk entsteht, das dadurch eher als Kompromiss erscheint denn als freie Darlegung eines autonomen intellektuellen Projekts (nach Art der creatio ex nihilo oder der unter kontingenten Umständen erfolgenden Offenbarung eines Werkes, dessen Notwendigkeit von aller Ewigkeit her gegeben war - wie es der »Idealismus« des Philosophen oder des Scholiasten unterstellt). Mauss erinnert im Vorwort zu Le Socialisme an Durkheims Interesse am Sozialismus, er stellt es in einen Zusammenhang mit den theoretischen Fragestellungen und dem ethischen Interesse des Soziologen und beschreibt schließlich das Verhältnis des Professors zu einem heterogenen Publikum, das einerseits aus Vertretern der Universität (Moralphilosophen, 
Spiritualisten, liberalen oder christlichen Ökonomen) und andererseits aus Studenten mit Sympathien für die Sozialisten bestand:

„Er versuchte, Partei zu ergreifen und diese Parteinahme zu begründen. Dazu hatte ihn eine Reihe von Vorkommnissen veranlasst, die teils geringfügiger und persönlicher Art, teils aber auch gravierender waren. Er ärgerte sich über den Vorwurf des Kollektivismus, den ihm anlässlich seines Buchs Über soziale Arbeitsteilung einige empfindliche Moralphilosophen und mehrere klassische oder christliche Ökonomen gemacht hatten. Aufgrund solcher Gerüchte hielt man ihn von Pariser Lehrstühlen fern. Andererseits hatten sich einige seiner brillantesten Studenten zum Sozialismus bekehrt oder genauer zum Marxismus oder Guesditismus. In einem Arbeitskreis für soziale Studien kommentierten manche Das Kapital von Marx, wie sie Spinoza kommentierten. Durkheim spürte diese Abneigung gegen den Liberalismus und den bürgerlichen Individualismus «. ${ }^{25}$

Die soziologische Ausdrucksarbeit, eine theoretische Arbeit, wenn man so will, ist die Formulierung eines Anspruchs, der Versuch, die von Gruppen gestellten Fragen in eine Fragestellung zu integrieren, und dies in einem differenzierten Problemfeld, dessen Ausdrucksstrategien implizieren, dass man durch die Ablehnung bestimmter Positionen nicht in andere, von den Gegnern vorgezeichnete Strategien eingeschlossen wird. Neben dieses Ausdrucksspiel (innerhalb der Beziehungen zu den Kollegen und auf der Ebene bestehender Fragestellungen) tritt ein weiteres, im Verhältnis zu den Studenten (einem Publikum, das entweder in Gestalt von Neugier oder von Fragen Probleme einführt, die außerhalb der in der universitären Tradition behandelten Problemfelder liegen). Der Marxismus und ganz allgemein die sozialen Probleme und der Sozialismus standen damals in den 1880er und 1890er Jahren außerhalb der Theorien und Interessen, die zum anerkannten Kreis der akademischen Problemfelder gehörten. ${ }^{26}$ Der an der Universität arbeitende Soziologe hat hier auch die Aufgabe, diese Fragen durch entsprechende Umarbeitung in die Universitätsphilosophie einzubringen. Die von Mauss angesprochene Haltung, die dem Verhältnis des Propheten zu seinen Zuhörern im Sinne Webers ähnelt, erkennt man auch bei Durkheim, etwa wenn er am Schluss seiner Vorlesungen über den Sozialismus eine Diagnose der ideologischen Strömungen vorstellt und eine Theorie skizziert, indem er hier von einem sozialen Bedürfnis spricht, dessen wahre Bedeutung die Sozialwissenschaft zum Ausdruck bringen müsse. Vor allem seine Interpretation der kurz zuvor im intellektuellen Leben aufgetauchten neoreligiösen Strömung zeigt, dass Durkheim die Soziologie auch als Versuch versteht, jene Denkweisen und Meinungen $\mathrm{zu}$ identifizieren und $\mathrm{zu}$ charakterisieren, die er anderswo (vor allem in den Regeln) als »freie Strömungen des kollektiven Lebens" bezeichnet. "Es gibt frappierende Übereinstimmungen [...] zwischen der Zeit, die wir untersucht haben (der des Saint-Simonismus) und der Zeit, in der wir leben, folgende drei Ideen sind hier gleichzeitig aufgekommen: die Idee, die Methoden der positiven Wissenschaften auch auf die Sozialwissenschaften auszudehnen [...]; die Idee einer religiösen Erneuerung; und schließlich die sozialistische Idee. ${ }^{27}$ Durkheim identifiziert diese drei Entwicklungen und sieht in ihrer Verbindung (ihrem gleichzeitigen Aufkommen zu seiner Zeit) und ihrer tiefgreifenden Konvergenz (jenseits der oberflächlichen Widersprüche) ein Kennzeichen der Krise der modernen Gesellschaften und der Bemühungen, einen Ausweg aus dieser Krise zu finden (was dann gelingen kann, wenn man dafür sorgt, dass sie zusammenfließen statt sich in unterschiedliche Richtungen zu bewegen). "Unser Schluss lautet also, wenn wir mit all diesen praktischen Theorien, die seit dem Beginn des Jahrhunderts keine sonderlichen Fortschritte gemacht haben, einen Schritt 
vorankommen wollen, müssen wir uns ganz methodisch darum bemühen, diese verschiedenen Bestrebungen zu berücksichtigen und nach ihrer Einheit zu suchen. ${ }^{28}$

Die Arbeiten von J.-C. Filloux und B. Lacroix können das Verdienst beanspruchen, entgegen dem Bild der Kälte des Positivisten oder der Zwanghaftigkeit des Neuscholastikers an die emotionalen Momente des wissenschaftlichen und ethischen Unternehmens Durkheims zu erinnern - wobei das psychoanalytische Interesse ihre Aufmerksamkeit für diesen Aspekt der Frage zweifellos geschärft hat. Aber nicht hier liegen unsere Streitpunkte. Sie betreffen vielmehr den Inhalt, die Bedeutung und den Kontextbezug dieses Prophetentums. Die unter dem Banner der Psychoanalyse mit leichter Hand vorgenommene Analyse erlaubt es, auf eine soziologische Analyse der intellektuellen und sozialen Konstellation zu verzichten, in der sich Durkheims Soziologie entwickelt. Die oben angesprochenen Illusionen haben System. Die Illusion des Schöpfers als Monade gestattet es, bei der Betrachtung eines geistigen Schöpfers von dem intellektuellen Milieu zu abstrahieren, aus dem er seine Anregungen, seine Vorbilder, aber auch seine Hemmnisse bezieht, und die Denktraditionen außer Acht zu lassen, von denen her er (im Widerspruch oder mit Sympathie) seine eigenen Fragestellungen erfindet ${ }^{29}$ - eine Sonderform dieser Illusion besteht darin, die Austauschbeziehungen innerhalb einer intellektuellen Gruppe oder Schule $\mathrm{zu}$ vernachlässigen, mit den unmittelbaren Vorgängern und den Weggefährten einerseits, den Mitarbeitern und Schülern andererseits. Man wird zugeben müssen, dass dies in Durkheims Fall besonders unangemessen ist, dessen Unternehmen schon sehr bald untrennbar mit der Organisation und Strukturierung eines Gemeinschaftsprojekts im Umkreis der Année sociologique verbunden war. Da es sich um die Entwicklung eines wissenschaftlichen Problemfelds in den Arbeiten der Mitglieder einer Gruppe handelt, wird man hinzufügen müssen, dass die Betrachtung der Entwicklungen der Durkheimschen Soziologie in den Arbeiten von Bouglé, Davy und Mauss vor allem im Blick auf das Thema, das uns hier interessiert ${ }^{30}$, eigentlich die Möglichkeit hätte eröffnen können, eine präzisere und besser begründete Antwort auf die Frage nach der Stellung und dem Inhalt einer politischen Soziologie zu geben. Die Illusion der existentiellen Krise als Auslöser (wie man sie auch bezeichnen mag, als »Neurose« zum Beispiel, um den bestechendsten Tendenzen nachzugeben, sofern man den Psychojargon nicht scheut) verstärkt noch den schematischen Charakter der Analyse: Man nimmt einen Urkonflikt, der sich erst im Verlauf einer Biographie entfaltet, gegebenenfalls mit der einfachen Peripetie einer zentralen Krise, in der der Konflikt (für den Analytiker) erkennbar wird und sich dauerhaft verknotet, um dann ins Werk einzufließen. Um die Rechte der historischen Erklärung gegenüber diesem psychologischen Essentialismus zu wahren, könnte man wenigstens verlangen, dass man, wenn die emotionale Last auf diese erste Krise zurückgeht, einräumt, dass diese ursprüngliche psychologische Struktur, die zweifellos Einfluss auf die Geschichte der betreffenden Person hat, als Hintergrund begriffen wird, vor dem die verschiedenen Ereignisse, die die betreffende Biographie ausmachen, sich abgespielt und ihren Einfluss geltend gemacht haben (die Familiengeschichte, die Geschichte der beruflichen Laufbahn, die intellektuelle Geschichte, die politische und die soziale Geschichte, denn es gibt auch eine Geschichte und nicht nur Wiederholungen der Urszene); dass man einräumt, dass es sich nicht um einen unveränderlichen Monolithen handelt, sondern um eine Konstellation, die (zumindest in ihren Ausdrucksformen) Veränderungen zulässt. Mehr als das brauchte der Soziologe nicht, um dort, wo eine bestimmte psychoanalytische Rekonstruktion (oder sollte man in einem etwas anderen Sinne 
sagen: ein Familienroman?) ein solipsistisches Drama zeichnet, den Schatten der Geschichte, eine kollektive Geschichte und keine einzelne Lebensgeschichte $\mathrm{zu}$ erkennen. ${ }^{31}$

Aus der Sicht der intellektuellen Biographie setzt sich diese Illusion in einer weiteren fort oder nimmt darin spezifische Züge an, einer Illusion, die einen bestimmten Zeitpunkt für die Konstituierung der Fragestellung definiert, mit Vorliebe die Jugendoder Bildungsjahre, wobei dieser ursprüngliche Entwurf in der Folge nur noch unbedeutende, den Umständen geschuldete Abwandlungen erfahren haben soll. Nun setzt aber eine Geschichte von Werken und geistigen Schöpfern die Erkenntnis voraus, dass Konstellationen keine schlichten Peripetien oder bloßes Dekor sind, vor deren Hintergrund eine von Anfang an in der Zelle der Familie vorbestimmte Geschichte abläuft. Die Umgestaltungen des wissenschaftlichen Projekts erfolgen im Zuge der intellektuellen und akademischen Laufbahn, in Abhängigkeit von der globalen Entwicklung des universitären und des intellektuellen Felds. Die »Rückkehr des Religiösen « in Durkheims wissenschaftlichen Interessen, um hier nur dieses Beispiel zu nennen, bedeutet nicht einfach nur die (in ihren Umständen kontingente und nur in ihrem Ursprung determinierte) Wiederkehr eines ursprünglichen väterlichen Projekts für Emile Durkheim; sie erhält ihre Bedeutung erst innerhalb einer bestimmten intellektuellen (neoreligiöse Bewegung), politischen und sozialen wie auch akademischen Konstellation. Eine Biographie bedarf also auch einer zeitlichen Gliederung im Blick auf die Periodisierung des geschichtlichen Feldes, das ihr Umfeld bildet (eines akademischen Milieus, einer politischen Geschichte, einer Sozialgeschichte).

Diese miteinander verwandten Illusionen gehören zu einer essentialistischen und ahistorischen Sicht der geistigen Produktion. Sie werden erleichtert durch gewisse Verzerrungen der hermeneutischen Methode, von denen oben bereits die Rede war (Willkür der Interpretation, Pointillismus der Zitate) und durch bestimmte Verwendungsweisen der biographischen Methode, die den geistigen Schöpfer isolieren und den Biographen dadurch zu einer unkontrollierten Autonomisierung verleiten. Eine sicherere und zugleich wohl auch ökonomischere Methode besteht darin, die Biographie vor dem Hintergrund einer Soziographie des Milieus, der Schule oder der Berufskollegen, kurz, eines Kollektivs zu konstruieren, dessen Tableau die Möglichkeit eröffnet, den betrachteten Fall als spezifische Konfiguration von Variationen $\mathrm{zu}$ begreifen, deren Gesamtspektrum die untersuchte Population bietet. Für die Biographie ist es in diesem Sinne von Vorteil, sich - um es mit den Worten der Althistoriker zu sagen - auf eine Prosopographie zu stützen.

Das erste Problem, vor das man sich gestellt sieht, wenn man Durkheims intellektuelle und soziale Entwicklung und den Problemkontext seiner Soziologie untersuchen möchte, ist das der Stellung des "institutionellen Prophentums", die der Soziologe einnimmt. So könnte man eine ganze Reihe von Aspekten der intellektuellen Bemühungen Durkheims bezeichnen, denn J.-C. Filloux und B. Lacroix werden durchaus dem Reichtum seines wissenschaftlichen Projekts gerecht, auch wenn sie sich auf die unterstellten persönlichen und psychologischen Ursprünge und auf eine sehr abstrakte Charakterisierung dieses Projekts beschränken - Filloux auf den Moralphilosophen, Lacroix auf den Gründer einer Wissenschaft, wobei der eine auf das Stereotyp des abgewiesenen jüdischen Propheten zurückgreift, der andere auf den aus der Epistemologie der Leser von Canguilhem und Althusser übernommenen Erbauer 
wissenschaftlicher Reiche. Diese Charakteristiken lassen sich allerdings nicht allein aus der Biographie dieses einzelnen Menschen erklären (ein auf politische Reform und die Gründung einer Wissenschaft abzielendes Projekt, das ein junger Intellektueller sich im Augenblick einer politischen Krise vornimmt; ein auf die Moral gerichtetes Projekt, das die ererbte religiöse Berufung fortführt und verlagert - »man darf nicht vergessen, dass ich der Sohn eines Rabbiners bin«). Wer den Vorwurf des Reduktionismus vermeiden möchte, den die eifersüchtigen Hüter der unantastbaren Rechte der Subjektivität gegenüber jeglichem Versuch einer rationalen Analyse wissenschaftlicher oder künstlerischer Hervorbringungen erheben, der sich nicht damit begnügt, ein bloßer Begleitdiskurs oder bloße Begleitmusik eines aufgeklärten Konsums zu sein, verfällt leicht in eine Abstraktion - die singularisierende biographische Abstraktion, die das Gegenstück zur reduktionistischen Abstraktion bildet. Zweifellos werden nicht alle laisierten Söhne von Rabbinern Durkheims, um Sartre zu paraphrasieren, doch dieser Sohn eines Rabbiners teilte mit anderen Juden nach der Assimilation den für die Dritte Republik grundlegenden bürgerlichen Optimismus, den Glauben an die Notwendigkeit und den Wert einer laisierten Moral; mit anderen an der Universität teilte er den Glauben an die Wissenschaft, mit anderen (und gegen wiederum andere) Philosophen die Überzeugung, dass man in bestimmten, bislang von einer spekulativen Philosophie beherrschten Bereichen ein positives Wissen schaffen könne; mit anderen Philosophen und Intellektuellen das Gefühl, dass die politischen und sozialen Fragen nach einer philosophischen Antwort verlangten und dass jene Reihe von Problemen und Diskussionen, die durch die Ausdrücke "soziale Fragen" und "Sozialismus" abgedeckt wurden, in die Philosophie eingebracht werden sollten - eine thematische Öffnung und Erneuerung, die das Eindringen der Probleme von nichtcousinschen Philosophen, die außerhalb der Universität standen, in die alten Fragestellungen der Universitätsphilosophie signalisiert. Man könnte diese Fragestellungen bis ins Unendliche erweitern - in konzentrischen Kreisen, wie sie an der Wasseroberfläche entstehen, wenn man einen Stein in einen Teich wirft. Zumindest muss jedoch daran erinnert werden, dass die Bemühung um eine rationale Antwort auf die politischen Fragen, die bereits eine lange Vergangenheit hat (mindestens seit der Revolutionszeit, dem Niedergang der religiösen Autorität und dem in diversen Formen erfolgten Aufstieg eines Mythos von der Macht des Geistes ${ }^{32}$ ) - dass diese Bemühung präzisere Formen annimmt und ein für ihre Entfaltung günstiges Umfeld findet mit der Krise der alten politischen Eliten aufgrund der Entlassung des kaiserlichen Personals und vor allem nach dem 16. Mai der Entlassung der alten Würdenträger und der mit der alten Macht verbundenen Teile der Beamtenschaft ${ }^{33}$, und dass sie sich vor allem in Louis Liards Projekten und Reformen mit den Bemühungen um eine erneuerte Universität trifft. Louis Liard, Leiter der Abteilung Höheres Bildungswesen im Bildungsministerium und "Protektor" der beruflichen Karriere Durkheims in deren Anfangsphase, institutionalisiert die Sozialwissenschaft auf der Grundlage einer Krise der spiritualistischen Universitätsphilosophie und deren Unvermögen, den Ansprüchen einer positiven Politikwissenschaft zu genügen. Die politische und soziale Prophetie, mit dem Anspruch des inspirierten Dichters (romantischer Mythos des Dichters) und mit dem Monopol auf die Stellung des abweichenden politischen und religiösen Reformers (die sozialistischen Reformer und letztlich Auguste Comte), wird für einen Teil dieser Generation (die positivistische und republikanische Gruppe) zur Aufgabe des Hochschullehrers. Zugleich eröffnet die Krise in der Einpflanzung der religiösen Moral (verbunden mit der Herausforderung der katholischen Kirche durch Positivisten und 
Republikaner) ihnen im Blick auf das Volk eine neue Rolle als »Prediger«. Diese neuen Funktionen überdecken das alte Bild des gebildeten Redners, das durch das politische und militärische Scheitern des Zweiten Kaiserreichs in Misskredit geraten ist, aus dem im universitären Bereich das Scheitern der Cousinschen Spiritualisten und der Moralphilosophen wird. ${ }^{34}$ Dies allerdings im Rahmen einer Konkurrenz der Fachgebiete um diese Funktion der politischen Beratung und der Moralisierung des Volkes. Wenn die Cousinschen Spiritualisten in Misskredit geraten (und gewissermaßen out sind), bleiben noch die Historiker und die Soziologen. Die Konkurrenz zwischen diesen beiden Berufsgruppen ist nicht nur epistemologischer Art. Es gibt auch eine Konkurrenz um die Stellung des Moralvermittlers und Volkspredigers, die durch die Organisation und Institutionalisierung eines neuen, auf das Volk ausgerichteten Unterrichts bedeutsam wird.

Das Prophetentum ist daher nicht allein das Produkt einer einzelnen psychologischen Transaktion. Wenn in der Unterbrechung der Familientradition (der Nachfolge im Amt des Rabbiners) für Durkheim ein psychologisches Problem liegt, so gibt es eine institutionelle Konstellation, welche Lösungen vorzeichnet, die nicht allein das Ergebnis einer wahnhaften Projektion sind. Und wenn es eine prophetische Versuchung als Antwort auf ein individuelles Problem gibt, so erklärt sich der spezielle Charakter dieses »institutionellen« Prophetentums durch den sozialen Kontext, in dem es entsteht.

\section{Die Wahl des Gegenstands: Taxonomien und Strategien}

24 Hat Durkheims Soziologie eine politische Zielsetzung? Räumt sie der Politikwissenschaft einen Platz ein? Behauptet sie, eine Antwort auf oder - in Gestalt einer Moral - ein Mittel gegen eine politische Krise bereitzustellen? Wir werden diese Fragen auf dem Umweg - oder vermittelt - über andere Fragestellungen angehen: erstens die Frage der existentiellen und geschichtlichen Genese der Durkheimschen Fragestellung (mutmaßlich die politische Krise der Dritten Republik); zweitens die nach der Stellung einer politischen Soziologie innerhalb der Durkheimschen Soziologie (insbesondere die Frage nach der Interpretation der Physik der Sitten und des Rechts); drittens die Frage nach Durkheims "Politik» (explizite oder diffuse politische Stellungnahmen; im Werk implizit enthaltene politische Theorie; Reichweite und politischer Status der Soziologie und letztlich die Bedeutung einer Prophezeiung auf soziologischer Grundlage; die Beziehungen zwischen dem wissenschaftlichen Werk und den zugehörigen politischen Fragestellungen während seiner beruflichen Laufbahn).

Hieße es nicht, allzu sehr Sartres Bild des Intellektuellen zu folgen (man denke etwa an das Nizan-Porträt in der Einleitung zu Aden), wenn man in den akademischen Entscheidungen des jungen Durkheim Reaktionen auf eine unmittelbare und direkt empfundene politische Situation erblickte? Hier sei zunächst an ein paar Daten und Fakten erinnert. Die Zeit der "vorläufigen Festlegung" seines akademischen Projekts, die seines Studiums an der Ecole normale supérieure in den Jahren 1879-1882, folgt unmittelbar auf eine politische Krise: die stockende Einführung des republikanischen Regimes und der republikanische Sieg nach der Krise des 16. Mai 1877. Aber kann man sagen, der junge Mann habe diese Krise in einer einsamen Reflexion wahrgenommen? Kann man sagen, sie sei ausschließlich politischen (oder auch nur konstitutionellen 
und parlamentarischen) Charakters? Die Krise ist nicht allein politischer Natur. Sie ist zugleich auch sozialer Natur, und das in zweierlei Hinsicht: Sie ist eine Krise, die mit den Klassengegensätzen zusammenhängt, und zugleich die tiefere, der erstgenannten zugrunde liegende Krise einer Gesellschaft, deren alte (auf verworfenen Absolutheitsansprüchen, religiösem Glauben, königlichem Absolutismus oder Despotismus basierende) Ordnung zusammengebrochen, aber nicht durch eine neue, auf neuen Prinzipien basierende Ordnung ersetzt worden ist. Die schwierige Anfangsphase der Republik nach dem Zusammenbruch des Kaiserreichs weckt genau die Fragen, die aus dem langen Nachdenken über die Krise der französischen Gesellschaft seit der Französischen Revolution hervorgegangen sind. Durkheim denkt, wie die übrigen jungen Intellektuellen seiner Generation, nicht allein, und er denkt nicht ausschließlich im Blick auf die unmittelbar aktuellen Peripetien. Es gibt eine philosophisch-historische Tradition der Reflexion über die soziale Krise in der doppelten Bedeutung dieses Ausdrucks. Vor allem Renan (La réforme intellectuelle et morale en France, 1871) und Taine (Les origines de la France contemporaine, 1875-1983) stecken den Rahmen dieser Reflexion ab. Über sie denkt man unwillkürlich auch an einen Einfluss Tocquevilles oder zumindest an die Tradition der liberalen Überlegungen zum Zerfall der organischen Gesellschaft des Ancien Régime, auch wenn solche Einflüsse nicht klar belegt sind, weder in den Studien zu Durkheims intellektueller Biographie noch durch Verweise auf diese Denktradition in Durkheims Werk. Man kann sich vorstellen, dass diese Tradition des politischen Denkens vor allem über Fustel de Coulanges (einen von Durkheims Lehrern an der Ecole normale) zu Durkheim gelangte, aber allgemeiner noch und weniger philosophisch durch die Kritik an der Romantik, die damals im Literaturunterricht weit verbreitet war. Eine optimistische laizistische und demokratische Tradition, die die Befreiung des Einzelnen aus der Knechtschaft der alten Ordnungen und das Verschwinden der alten, nicht durch die Vernunft begründeten Autoritäten feierten, eine Tradition, zu der Durkheim vor allem über Renouvier Zugang findet, verbietet es auf der anderen Seite, in der Befreiung des Einzelnen und der Forderung nach einer gesellschaftlichen Befreiung Zeichen des Zerfalls eines organischen Gesellschaftszustands zu erblicken. Vielmehr ist daran zu erinnern, dass die positivistischen Themen in der popularisierten Form, in der sie in den 1870er Jahren verbreitet wurden, den Pessimismus eines Zerfalls der sozialen Ordnung mit dem Voluntarismus der Notwendigkeit eines Wiederaufbaus und dem Optimismus der Aufklärung verbinden, vor allem über die Hoffnungen, die man in die Bildung legt (zugleich im Sinne von Ausbildung und im Sinne der Einpflanzung einer neuen Moral).

Im Übrigen lauert im Bereich des Durkheimschen Verhältnisses zur Politik auch die Gefahr des Anachronismus. Wir dürfen nicht übersehen, welche Trägheit Denkmuster und Vorstellungen besitzen können. Vor allem in Über soziale Arbeitsteilung verdanken die Metaphern der Trennung und des Krieges zwischen den Klassen viel den Darstellungen aus der römischen Geschichte (Auszug der Plebs, Sklavenaufstände). Man vergisst allzu leicht, welche Bedeutung diese Vorbilder einer Interpretation der politischen und sozialen Krise für ein politisches Denken haben konnten, das von der kaiserlichen Zensur zu historischen Vergleichen gedrängt wurde und sich von der prägnanten griechisch-römischen Kultur leiten ließ. Die Geschichte der Antike funktioniert nicht nur wie ein Satz Schlüssel, sie legt auch Deutungsmuster für die Interpretation der Situation und der Krise nahe. Außerdem bildet sie allein aufgrund ihrer Funktion als Mittlerin und als Projektionsfläche einen Faktor, der das 
»Mysterium« der Krise verstärkt, und sie erleichtert Übertragungen: Der mit antiken Metaphern maskierte Krieg zwischen den Klassen ist das einfache Symptom einer weitaus tieferen "gesamtgesellschaftlichen" Krise, wie man nach heutigem Sprachgebrauch sagen könnte. Dadurch wird es möglich, in der Interpretation der Krise der modernen Gesellschaft von einem Modell des Klassenantagonismus (sagen wir, einem marxistischen Modell) zum Modell einer Krise der Einheit und Integration eines Organismus überzugehen - entweder, auf der Ebene der Metaphern, zu organizistischen Deutungsmustern, oder, auf der der Ebene der Diagnose der Ursachen dieses Übels, zu einer Interpretation, die den Akzent auf die Krise der gemeinsamen Glaubensüberzeugungen, des Konsenses, legt. Die antike »Lesart« der modernen Krise ist nicht nur Ausdruck einer dekorativen Verwendung der althistorischen Kenntnisse oder der Überrest (rhetorische Trägheit) einer (aus der kaiserlichen Zensur erwachsenen) Gewohnheit, in der politischen Philosophie die Dinge nur auf Umwegen und verdeckt zu sagen (die Kritik kommt unter der Maske antiker Beispiele daher). Die Akteure einer Situation reichern diese bekanntlich, wie Marx angemerkt hat, häufig mit Erinnerungen an eine vergangene Situation an, vor allem wenn sie als Schüler eines Bildungssystems, das sie mit der griechisch-römischen Kultur getränkt hat, große Distanz zur historischen Gegenwart empfinden. Es ist ein Unterschied, ob man das historische Geschehen unmittelbar beobachtet oder ob man es solcherart über Plutarch, Titus Livius oder Tacitus vermittelt betrachtet. Die durch solche Deutungsmuster verursachte Unschärfe entspricht auch einer Notwendigkeit. Es ist frappierend, wie sehr die soziale Krise im politischen Diskurs der 1870er und 1880er Jahre zugleich präsent ist und versteckt wird, oft unter dem Bild der politischen und der moralischen Krise. Hier stellt sich die Frage nach der Distanzierungswirkung solch einer Lesart der Geschichte, vor allem auf einen Mann wie Durkheim. Gewöhnlich gilt die Zugehörigkeit zu einer (ethnischen, kulturellen, religiösen) Minderheit nach einer etwas kurz greifenden Philosophie des Unterschieds und der Marginalität als günstige Voraussetzung für die Berufung zum Soziologen. Mir scheint, es gibt möglicherweise wichtigere Gründe für dieses grundlegende und ursprüngliche "Fremdsein« als Voraussetzung soziologischen Fragens. In Durkheims Fall könnte man sie bei der distanzschaffenden Wirkung der Vielfalt und Überlagerung kultureller Deutungsmuster der Geschichte suchen (jüdische Kultur, griechisch-römische Kultur). Diese »Lesarten « tragen oder suggerieren auch Diagnosen. Insbesondere stellt sich die Frage, ob die Interpretation auf der Grundlage des religiösen und die Deutung auf der Grundlage des moralischen Faktors in diesen kulturellen Bezügen nicht zur Deckung gelangen, weil sie tief in den historischen Metaphern präsent sind. Wir werden gleich darauf zurückkommen.

Wir haben auf einige der Vermittlungsglieder hingewiesen, die zwischen einem jungen Universitätsangehörigen und der von ihm wahrgenommenen politischen Landschaft stehen mögen. In der Tat müssen wir uns vor dem allzu einfachen, aus optimistischen und naiven Vorstellungen von "Engagement « stammenden Bild eines unmittelbaren Kontakts zwischen dem Bewusstsein des (politischen oder soziologischen) Theoretikers und der "Geschichte« hüten - als böte sich die Geschichte dem Blick vollkommen klar und ungetrübt wie eine gänzlich überschaubare, in guter Sichtweite liegende Landschaft dar. Die unterschiedlichen Vermittlungsglieder - Echos der geschichtlichen Veränderungen der französischen Gesellschaft in der philosophischen und historischen Reflexion, übersetzt gemäß den ererbten Rastern des historischen und moralischen Studiums der griechisch-römischen Gesellschaften - fungieren als 
Vermittlungssysteme, die die Wahrnehmung zugleich ausrichten, verfeinern und verzerren. Zur Charakterisierung dieser Effekte könnte man auf diverse Bilder zurückgreifen, etwa auf das des Überdruckens oder das des Tilgens. Dieses Modell der zugerüsteten und vermittelten Wahrnehmung findet seine Rechtfertigung nicht nur in den allgemeinen Merkmalen der - vor allem - geschichtlichen Wahrnehmung, sondern in diesem Fall auch in der Tradition der verzerrten, aus dem Erbe der politischen Zensur des Zweiten Kaiserreichs und der Abschließung der akademischen Welt gegenüber aktuellen politischen und sozialen Fragen hervorgegangenen Wahrnehmung. $\mathrm{Zu}$ den Schirmen, die zwischen der Wahrnehmung des jungen Universitätsangehörigen und der historischen Situation stehen, muss man auch die historische Situation im Ausland zählen. Durkheims Studienaufenthalte in Deutschland gelten gemeinhin als Gelegenheit zur Entdeckung (oder vertieften Wahrnehmung) der Denktradition des Kathedersozialismus, der Universitätsorganisation, der Entstehung aus der Philosophie hervorgegangener positiver und experimenteller Fachgebiete (Psychophysiologie) und schließlich auch als Gelegenheit zum Kontakt mit bestimmten Strömungen des sozialistischen Denkens (insbesondere dem marxistischen Denken). Allerdings ist auch anzumerken, dass der junge Durkheim eine ideologische Konstellation und - durch sie übersetzt - eine soziale Konstellation entdeckt, die sich seiner Wahrnehmung der geschichtlichen Situation Frankreichs überlagern wird. Genauer gesagt, sein Deutschlandaufenthalt 1886 bietet ihm möglicherweise Gelegenheit, Situationen und Probleme kennenzulernen, die in der historischen Konstellation der 1890er Jahre in Frankreich wieder hervortreten (das Problem des Verhältnisses zum Sozialismus und des in den Zielsetzungen der Sozialisten erkennbaren Konflikts zwischen einer auf Organisation und Planung ausgerichteten Strömung und einer Strömung, die den Klassenkampf sowie Darstellung und Organisation der sozialen Antagonismen in den Vordergrund stellt): Die Schirme fungieren auch als Vermittler und als Organisationsrahmen für das Denken.

Selbst in Durkheims allerersten Schriften ist leicht zu erkennen, dass die politischen und sozialen Peripetien über ihr Echo in der politischen Philosophie Eingang in das Denken des jungen Universitätsmitglieds finden. Die - klassische - Reflexion etwa über die Erarbeitung einer Bestimmung des Gemeinwohls durch den Wettbewerb und die Konvergenz individueller Entscheidungen der politischen Subjekte findet ihre Aktualisierung und ihren Platz in politischen Debatten über das allgemeine Wahlrecht und die politische Beteiligung des Volkes, denen Durkheim in der Besprechung eines Buchs von Fouillée begegnet. ${ }^{35} \mathrm{Kommt}$ dieser verdeckten und verschleierten Präsenz der Politik in Durkheims Werk zentrale Bedeutung zu? Hier müssen wir auf die Bedeutung der Physik der Sitten und des Rechts und auf die der hypothetischen Präsenz einer politischen Soziologie in der Durkheimschen Soziologie (im engeren wie im weiteren Sinne) und ergänzend dazu auf die Frage nach Durkheims "Politik« eingehen, das heißt auf die Frage nach seinen politischen Stellungnahmen und seinen expliziten oder diffusen, direkten oder indirekten (von einzelnen Mitgliedern der Schule vermittelten) praktisch-politischen Vorschlägen.

Man kann problemlos erkennen, dass die Physik der Sitten und des Rechts, posthum herausgegebene Vorlesungen ${ }^{36}$, auf die Formulierung einer Moral zielen und keinen systematischen Entwurf einer Politikwissenschaft darstellen. Aber da Durkheim in diesem Text am explizitesten über den Staat und die Korporationen spricht, ist auch klar, dass sich dort eine »Politik« finden lässt, die in seinem übrigen Werk diffuser und verdeckter zum Ausdruck kommt. Wir werden kurz den Status der politischen 
Begriffsbildung in Durkheims Soziologie erörtern und dann ausführlicher der inhaltlichen Frage nachgehen, welche Politik in dieser Soziologie präsent ist, welche Fragen sie einbrachte und welche Veränderungen in Durkheims Fragestellung sie bewirkte. Die Physik der Sitten und des Rechts sind nicht die ursprüngliche Form einer politischen Wissenschaft, die durch die Umstände und Bedingungen der Weitergabe und Tradierung des Werkes verdunkelt worden wäre. Die Vorlesungen sind Teil eines allgemeinen, auf die Moral zielenden Projekts. In seiner Einführung zu dem von ihm 1920 herausgegebenen Manuskript, das die Einleitung zu diesen Vorlesungen werden sollte ${ }^{37}$, weist Mauss darauf hin, dass Durkheim eine Moral verfasst hatte, ein systematisches Werk, von dem man noch einige Elemente in Texten über die Familie (häusliche Moral) und über Berufsgruppen (Berufsmoral) finden könne. Durkheims Exposé unterscheidet zwischen einer universellen Moral, die im Verhältnis des Individuums zu sich selbst wie auch zu den anderen Menschen "ungeachtet jeder Gruppenzugehörigkeit« Geltung besitzt und auf Respekt oder die Entwicklung der Menschheit zielt - und diversen Formen einer "partikularen Moral«, die Regeln in Bezug auf die Stellung innerhalb einer Gruppe definieren: »häusliche Moral«, »staatsbürgerliche Moral«, »Berufsmoral«. ${ }^{38}$ Im Zusammenhang mit der staatsbürgerlichen Moral skizziert Durkheim eine Theorie des Staates und insbesondere des Verhältnisses zwischen Staat und Gesellschaft. Aber nichts weist darauf hin, dass man darin den Entwurf einer politischen Wissenschaft erblicken müsste, deren Projekt Durkheims gesamten wissenschaftlichen Bemühungen die Richtung vorgäbe.

Durkheim hat verschiedene Klassifikationen der Soziologie vorgelegt, ob nun in Gestalt programmatischer Tableaus oder systematischer Bilanzen des Fachgebiets. Hier kann man insbesondere auf "Les études de science sociale ${ }^{39}$ verweisen; des Weiteren auf einen erstmals in italienischer Sprache erschienen Aufsatz über den »aktuellen Stand der soziologischen Forschung in Frankreich « $(1895)^{40}$, auf das Vorwort zum ersten Band der Année sociologique (1898) ${ }^{41}$ und den Plan für diesen ersten Band, mit den im folgenden Jahr an diesem Plan vorgenommenen Veränderungen (der den systematischen Rahmen für die wissenschaftliche Arbeit Durkheims und seiner Gruppe darstellt und den man mit den späteren, von Mauss vorgeschlagenen und kommentierten Veränderungen vergleichen könnte), auf die Aufsätze »La sociologie en France au XIX ${ }^{e}$ siècle« $(1900)^{42}$, "La sociologie et son domaine scientifique« (1900) ${ }^{43}$ (hier könnte man auch den von Paul Fauconnet und Marcel Mauss verfassten Aufsatz "Sociologie« von 1901 einschieben ${ }^{44}$ ), "Sociologie et sciences sociales « (1903, zusammen mit Paul Fauconnet ${ }^{45}$, und ein weiterer Aufsatz mit demselben Titel aus dem Jahr $\left.1909^{46}\right)$ sowie $»$ La sociologie $(1915)^{47}$. In all diesen Texten, die zwischen dem zuweilen systematisierten Programm in Gestalt eines Forschungsplans und der Bilanz kritisches, geschichtliches, bewertendes Tableau - wechseln und die jeweils entweder mit einer Übersicht über das gesamte Fachgebiet oder einem bevorzugten Verweis auf die Durkheimgruppe verbunden sind (die sich mit der Zeit natürlich durchsetzt), in all diesen Texten findet sich in unterschiedlichen Graden der Explikation eine Taxonomie des Fachgebiets. Ohne ins Detail zu gehen, wollen wir uns hier bezüglich des Platzes oder des Fehlens - einer politischen Soziologie auf den Aufsatz von 1885, eine Buchbesprechung mit programmatischer Funktion, und den Plan für die Rubriken der Année sociologique mit seinen sukzessiven Veränderungen beschränken, einschließlich der nach Durkheims Tod vorgenommenen, was drei weitere Bezugszeitpunkte ins Spiel 
bringt: 1898, 1899 und 1927. Betrachtet man diese Texte in ihrer Abfolge, fällt ins Auge, dass die Politik zunehmend daraus verschwindet.

31 "Les études de science sociale«, Durkheims erster längerer Text nach den Buchbesprechungen isolierter Werke 1885, diskutiert nacheinander ein Buch von Spencer (den 6. Teil von Principles of Sociology über die kirchlichen Institutionen), ein Buch von A. Regnard (über den Staat), ein Buch von A. Coste, A. Burdeau und L. Anéat (Les questions sociales contemporaines) und ein Buch von Schäffle (Die Quintessenz des Sozialismus). Diese Zusammenstellung unterschiedlicher und »disparater« Werke bietet Durkheim die Möglichkeit, das Feld der Fachgebiete abzustecken, die in seinen Augen die wichtigsten soziologischen Wissenschaften darstellen. Er diskutiert nacheinander und nimmt sich dabei gegenüber den Büchern, von denen er ausgeht, mehr oder weniger Freiheiten heraus - die Religion (im Anschluss an Spencer), aber auch Moral und Recht als regulierende Instanzen (Recht, Moral, Religion "sind die drei großen regulierenden Funktionen der Gesellschaft $\aleph^{48}$ ); den Staat (und er ruft zur »Schaffung einer wissenschaftlichen Theorie des Staates « auf ${ }^{49}$, die reiner sein solle als die in Deutschland ausschließlich "von Philosophen und Juristen gepflegte» Staatswissenschaft); die politische Ökonomie, auf der Grundlage kritischer Arbeiten der orthodoxen liberalen Ökonomie und gestützt auf die deutsche Strömung einer Sozialökonomie. Diese Teildiskussionen führen ihn dazu, ein Organisationstableau der Soziologie vorzustellen, eine Unterteilung des Fachgebiets in verschiedene Zweige, eine innere Gliederung nach dem Vorbild der Wissenschaftsklassifikationen. Dort unterscheidet Durkheim drei "spezielle« soziologische Wissenschaften; "die eine untersucht den Staat, die andere die Regulierungsfunktionen (Recht, Moral, Religion), die dritte schließlich die ökonomischen Funktionen der Gesellschaft«. Diese Wissenschaften des Normalen finden ihr Gegenstück in einer Wissenschaft des Anomalen, der "pathologischen Soziologie, deren am weitesten fortgeschrittenen Teil die Kriminologie bildet« (und hier erwähnt er lobend die Aufsätze von Tarde in der Revue philosophique). Den speziellen soziologischen Wissenschaften steht schließlich eine allgemeine Soziologie gegenüber (nach dem Vorbild der Unterscheidung zwischen speziellen biologischen Wissenschaften und allgemeiner Biologie), zu der »die Arbeiten von Comte, Schäffle, Spencer, Lilienfeld, Le Bon, Gumplowicz, Siciliani usw. gehören« und deren Aufgabe es ist, »die Bildung des kollektiven Bewusstseins, das Prinzip der Arbeitsteilung, Rolle und Grenzen der natürlichen Selektion, die Gesetze der Vererbung oder der Kontinuität in der sozialen Evolution usw.« zu erforschen. ${ }^{50}$ Die Klassifikation arbeitet also mit der Unterscheidung zwischen Allgemeinem und Besonderem (übernommen oder wahrscheinlich eher autorisiert von der Unterscheidung zwischen allgemeiner Biologie und speziellen biologischen Fachgebieten oder - besser und in C. Bernards Kategorien - zwischen der allgemeinen Physiologie der Lebewesen und der Physiologie einzelner Arten); auf der Ebene der speziellen Fachgebiete mit der Unterscheidung zwischen Normalem und Pathologischem (ohne dass die Parallele zu weit getrieben würde) und auf der Ebene der Zweige mit der Unterscheidung zwischen Leitungsfunktion (Staat), Regulierungsfunktionen (Recht, Moral, Religion) und Verteilungsfunktionen (Ökonomie), die sich gleichfalls auf die Unterteilung der Physiologie berufen kann. Wie man sieht, befinden sich unter den speziellen Fachgebieten zwei, die bereits stark ausgebildet sind, nämlich die politische ökonomie (in ihrer sozialen und kollektiven Form, der Sozialökonomie, oder ihrer individuellen Form, der klassischen Ökonomie) und die Staatswissenschaft ${ }^{51}$ sowie schließlich eine dritte Disziplin, die weit weniger ausgebildet ist, die Erforschung der 
Regulierungsfunktionen - womit Durkheim das Feld bezeichnet, auf dem sich die Soziologie in erster Linie betätigen soll. Um den besonderen Charakter der Interessen der universitären Soziologen seiner Generation (Richards und seiner eigenen) im Gegensatz zu denen von Fouillée und Espinas zu kennzeichnen, weist er 1895 in einer Übersicht über das Fach und die zeitgenössischen Strömungen darauf hin, dass sie auf die Erforschung der allgemeinsten Phänomene (die Natur der Gesellschaften, das Evolutionsgesetz, das Verhältnis zwischen dem Sozialen und dem Biologischen) verzichten, um sich "auf die Erforschung der moralischen Phänomene zu beschränken « ${ }^{52}$ Hält man an den oben beschriebenen Unterteilungen fest, bedeutet dies zugleich die Aufgabe einer allgemeinen Soziologie (umfassende Entwicklungsgesetze) und - auf der Ebene der »speziellen soziologischen Wissenschaften « - die Konzentration auf die Wissenschaft der moralischen Regulierungen, mit Ausnahme der Ökonomie und der Staatswissenschaft.

Die Klassifikation, die der Année sociologique zugrunde liegt, bestätigt das Verschwinden der Politik als des spezifischen Gegenstands eines Zweigs der Soziologie. Im Vorwort zum ersten Band definiert Durkheim den Bereich, in dem die Soziologie ihren Platz finden und sich mit anderen Fachgebieten austauschen soll, indem er die wissenschaftlichen Felder benennt, denen die Soziologen ihren Stoff und ihre Analysen entnehmen müssen, um jene, wie man es nennen könnte, ursprüngliche Akkumulation von wissenschaftlichem Kapital $\mathrm{zu}$ bewerkstelligen, für welche die Année sociologique steht. ${ }^{53}$ Zur kritischen Lektüre und für Systematisierungsbemühungen werden dort an "Spezialgebieten«, über die die Soziologen informiert sein müssen, die "historischen und ethnographischen Schulen Deutschlands und Englands « ${ }^{54}$ empfohlen; und als Spezialgebiete, die an die Soziologie herangeführt werden sollten, die Geschichtswissenschaft - die von der Geschichtswissenschaft gesicherten und analysierten Tatsachen bilden die Grundlage für vergleichende soziologische Studien ${ }^{55}$ - und, so der Hinweis in einer Fußnote, die "wirtschaftliche oder moralische Statistik«. ${ }^{56}$ Wodurch offensichtlich zwei verschiedene Bereiche definiert werden: auf der einen Seite das Feld der sehr nahen Fachgebiete, die entweder als Hilfswissenschaften der Soziologie (Statistik) oder als komplementäre Wissenschaften verstanden werden können (Geschichtswissenschaft), die dazu aufgefordert sind, sich letztlich mit der Soziologie $\mathrm{zu}$ vereinigen (eine These, die Durkheim im Blick auf die Geschichtswissenschaft immer dezidierter vorbringen wird). Auf der anderen Seite das Feld der benachbarten Spezialdisziplinen, denen die Soziologie einen Teil ihrer Materialien entnimmt (da könnte man die Rechtsgeschichte nennen und zweifellos auch die Ethnographie oder die Religionsgeschichte). Auch hier wieder ist festzustellen, dass die politischen Wissenschaften oder eine Politikwissenschaft fehlen.

Die systematische Organisation der Soziologie, die im Plan der Rubriken der Année sociologique sichtbar wird, gehorcht dem allgemeinen Prinzip der Unterscheidung zwischen Allgemeinem und Besonderem: allgemeine Soziologe (1. Abteilung) und diverse, zum Teil bereits gut ausgebildete Spezialzweige (Religionssoziologie - 2 . Abteilung; Soziologie der Moral und des Rechts - 3. Abteilung; Kriminalsoziologie - 4. Abteilung; Wirtschaftssoziologie - 5. Abteilung; ab 1898 kommt noch als 6. Abteilung die soziale Morphologie hinzu; vielleicht muss man dazu die zur Rubrik »Diverses" gehörende Soziologie der Ästhetik rechnen, die zwar von geringerer Bedeutung ist, aber über einen klar definierten Objektbereich verfügt); während andere, weniger ausgebildete Zweige in einer 6. Abteilung zusammengefasst sind (bzw. in der 7., als die soziale Morphologie als zusätzliche Abteilung aufgenommen wird), entweder weil es 
sich um spezialisierte Forschungstraditionen handelt, die stark auf eine bestimmte Fragestellung ausgerichtet sind (Sozialanthropologie), oder weil es sich um Formen der Erkenntnis des Sozialen handelt, die kaum in Durkheims Fragestellung integriert sind (Demographie). Die Objekte der politischen Wissenschaft finden sich hauptsächlich in der Abteilung "Soziologie der Moral und des Rechts", nehmen dort allerdings keine bedeutende Stellung ein, da diese Abteilung im Wesentlichen Arbeiten zur Rechtsgeschichte, zur Geschichte der Moral und zur Ethnographie gewidmet ist (soziale Organisation, Familienrecht, Ehe, Privateigentum, Vertrag, Verantwortung usw.). So sehen wir für das Jahr 1898 eine Unterabteilung »Politische und soziale Organisation«, in der sich auch ein Kapitel mit der Überschrift "Politische Organisation" befindet; und für das Jahr 1900-1901 eine Unterabteilung (III) »Politische Organisation«, deren erster Aufsatz (A) den Titel »Allgemeine Staatstheorie« trägt. Wenn die Staatstheorie in der Abteilung "Soziologie der Moral und des Rechts« untergebracht wird, weist man sie jener Wissenschaft der Regulierungen zu, die im ersten von Durkheim skizzierten Tableau der Sozialwissenschaft das Gegenstück zur politischen Ökonomie und zur Staatswissenschaft bildete, und verringert dadurch ihre Bedeutung wie auch ihren speziellen Charakter. Wenig Platz also für eine politische Theorie in Durkheims Systematik und wenig unmittelbares Interesse bei Durkheim an politischen Themen in den Rubriken der Année sociologique. Verändert sich diese Stellung in der ab 1927 unter Leitung von Mauss veränderten Form der Zeitschrift? Aus Zeitgründen ist es hier nicht möglich, Mauss' Aufsatz »Divisions et proportions des divisions de la sociologie ${ }^{57} \mathrm{mit}$ Überlegungen zur Einteilung und Bedeutung der Rubriken in der Année sociologique die Aufmerksamkeit zukommen zu lassen, die er in unserer Erörterung eigentlich verdiente; hier sei nur angemerkt, dass dieser Text eine privilegierte Quelle für die Erforschung des Verhältnisses der Durkheimschen Soziologie zur Politik wäre. Der Text, der nach dem Krieg und den Schlägen, den er der Gruppe zugefügt hatte ${ }^{58}$, eine neue Folge vorstellt, ist zugleich auch eine Bilanz und eine Rückkehr zu dem konzeptionellen Rahmen, der den Aufbau der Durkheimschen Soziologie prägte, und dies in einer Konstellation, in der die Soziologie bereits tiefer verwurzelt ist (»in einer Zeit, in der unsere Studien populär sind«) und in der auch die politischen Wissenschaften durch die Ecole des sciences politiques gut etabliert sind, zu einer Zeit auch, als durch die jüngeren Adepten (Halbwachs, Simiand usw.) die Verbindung des Durkheimismus zum Sozialismus und zu den politischen Bewegungen der Linken enger sein dürfte. So bietet sich hier eine gute Gelegenheit, das Verhältnis der Soziologie zur politischen Theorie zu klären. Kurz gesagt, weist Mauss der "politischen und staatstheoretischen" Forschung ihren Platz in der Rubrik "Allgemeine Soziologie» sowie in denen der Rechts- und der Wirtschaftssoziologie $\mathrm{zu}^{59}$ und erkennt zugleich den ungewissen Status und die unzulängliche Stellung dieser Art von Analysen in der Année an. Er gibt Beispiele für politische Fragen, bei denen Soziologen behilflich sein können (indem sie "positive Studien « zu politischen Problemen beisteuern), doch bei diesen Beispielen handelt es sich in Wirklichkeit um sozialpolitische Probleme wie die "Sozialversicherungen« und die "Einwanderung". Am Ende des Textes greift er die Frage in einer systematischeren Erörterung des Verhältnisses der Soziologie zur Politik nochmals auf. Mauss erinnert dort an die Durkheimsche Unterscheidung zwischen Wissenschaft und Kunst und knüpft daran an: um der Soziologie ihre wissenschaftlichen Privilegien zu sichern (Recht auf Umwege und das Ausklammern praktischer Probleme), aber auch um der politischen Kunst ihre Besonderheit und ihre Würde zu belassen. Er geht jedoch noch ein wenig weiter in dem Bemühen, eine 
»soziale Funktion« der Soziologen zu begründen, und zwar mit deren Beitrag zur kollektiven Lenkung durch die Gesellschaft wie auch zu deren Entwicklung und Veränderung. So erklärt er, dass es möglich sei, »die Wissenschaft dieser Kunst zu betreiben $«^{60}$ - Definition einer politischen Soziologie, versehen mit einem Urteil über die Versuche, auf der Basis der Geschichte und der Beobachtung (im Unterschied zum Dogmatismus und Normativismus der klassischen Rechtswissenschaftler) eine Wissenschaft der "politischen Formen und Verfassungen « zu schaffen. ${ }^{61}$ Erst hier sehen wir also wirklich, als Krönung der Soziologie, die Idee einer politischen Theorie aufkommen, womit auch klar ist, dass sie im Korpus der Durkheimschen Soziologie bis dahin fehlte. Doch diese Einführung der Politik in die Soziologie, die im Umkehrschluss bestätigt, was wir über deren Stellung in Durkheims Entwürfen gesagt haben, geht nicht ohne einen Umbau des konzeptionellen Raums: Definition einer Ebene für den Eingriff des Soziologen und einer besonderen Ebene der »politischen Soziologie«. Auf die Definition des Eingriffs des Soziologen im Vergleich zu anderen "Spezialisten« der sozialen Kunst (Staatsbeamte, Juristen) und auf die spezielle Eingriffsebene der Soziologen im politischen Bereich, der einen anderen Bereich der Politik als den der Auseinandersetzungen zwischen den politischen Akteuren definiert, werden wir noch zu sprechen kommen.

\section{Politische Krise, soziale Krise, moralische Krise}

Wir können also aus dieser taxonomischen Diskussion festhalten, dass der Platz der Politik in dem systematischen Rahmen, den der Plan der Rubriken in der Année sociologique darstellt, ungewiss war. Insgesamt können wir feststellen, dass die Staatswissenschaft im Verhältnis zu den anfänglichen Entwürfen des jungen Durkheim (1886) verschwindet und nur in geringfügigem Ausmaß in den von Mauss vorgenommenen Veränderungen zurückkehrt - und zwar in Gestalt des Bemühens, die politischen Anwendungsmöglichkeiten von Ergebnissen der Soziologie aufzuzeigen. Doch Programme und Ankündigungen sind etwas anderes als die tatsächlichen Inhalte und Gegenstände des wissenschaftlichen Unternehmens. Wie behandelt Durkheim Gegenstände einer "politischen Wissenschaft" (Staat, Parteien usw.)? Man könnte sich auf einen weiteren Anachronismus einlassen und Durkheims Bücher mit dem Begriffsraster der heutigen Politikwissenschaften lesen: Finden sich dort Anfänge einer Reflexion über Legitimität und Macht, über den Staat, über Repräsentation, über die Strukturen der Parteien, über Mobilisierung und dergleichen? Es wäre der Versuch, Durkheim auf das Prokrustesbett Duvergers zu legen - wobei er die Füße verlöre, aber möglicherweise auch den Kopf. Ein nicht sonderlich interessantes Vorgehen und als Beweis ohne Wert: Man behält immer etwas zurück, wenn man ein Werk solcherart durch ein Sieb passiert, aber die Logik des Werkes geht verloren. Lässt man Detailbemerkungen und analytische Exkurse außer Acht, behandelt Durkheim spezifisch politische Gegenstände nur in wenigen Texten, mit Ausnahme der Physik der Sitten und des Rechts und Le Socialisme. Das letztgenannte Buch ist eine ideengeschichtliche Analyse, eine Studie über das Streben nach dem Sozialismus und über dessen Grundlagen in der Organisation der modernen Gesellschaft. Es gehörte ganz speziell in den Bereich einer Soziologie der politischen Parteien und der sozialen Bewegungen. Aber darauf werden wir noch zurückkommen. Durkheims präziseste Texte über den Staat und das politische System finden sich in der Physik der Sitten und des Rechts. Bernard Lacroix, der allzu sehr damit beschäftigt ist, eine Theorie des 
politischen Systems ausfindig zu machen, schenkt den dort enthaltenen Analysen des Staates und des Parlamentarismus seltsamerweise keine Beachtung. Er ist vollkommen beherrscht von dem Wunsch, etwas zu erhellen und theoretisch zu kommentieren, das letztlich nur eine aus der Biologie übernommene Metapher darstellt, die Idee des "Substrats«. Auf der Grundlage dieses und einiger anderer Begriffe (kollektive Vorstellungen, Regeln usw.) versucht er ein allgemeines Schema des politischen Systems in seinem Verhältnis zum sozialen System zu konstruieren, doch dieses Schema erscheint als eine künstliche Konstruktion, die angesichts des punktuellen und fragmentarischen Charakters ihrer Begründung in Durkheims Texten nur von seinem theoretischen Willen zusammengehalten wird. Es wäre interessanter gewesen, die Aufmerksamkeit auf die von Durkheim vorgelegte Analyse des modernen Staates und des Parlamentarismus zu richten. Denn dort findet man, in Durkheims Sprache (Verhältnis des Staates zum »kollektiven Bewusstsein«), die stark von biologischen Analogien durchsetzt ist (man denke insbesondere an das Bild des Gehirns als zentralen Steuerungsorgans), die Theorie oder die Idealisierung des republikanischen Parlamentarismus. Der Staat ist das denkende, aufgeklärte und rationale Bewusstsein der Gesellschaft (der Staat ist »nur der Sitz eines speziellen, beschränkten Bewußtseins, das jedoch schärfer und klarer ist und eine lebendigere Vorstellung von sich selbst besitzt ${ }^{(62}{ }^{2}$. Hier ist nicht der Ort, um die Stellung dieser Analyse des Staates innerhalb des Durkheimschen "Systems" oder dessen Quellen zu untersuchen. ${ }^{63}$ Hier sei nur wiederholt, dass sie keine zentrale Bedeutung für Durkheims Soziologie besitzt und in den Durkheimschen Arbeiten - trotz gewisser Analysen von Bouglé und Mauss - keinen nennenswerten, der politischen Soziologie zugehörigen Bereich eröffnet oder vorbereitet. ${ }^{64}$

Von zentralerer Bedeutung für die Durkheimsche Soziologie sind zwei andere Gruppen von Bemerkungen. Die erste ist die Analyse der Funktionen des Staates als Schutz vor dem Unterdrückungspotential der beschränkten Gruppen, denen der Einzelne angehört (Sekundärgruppen, sagt Durkheim, weil zweitrangig und untergeordnet im Verhältnis zu der umfassenden sozialen Einheit, in der sie vereint sind). Mit dieser Auffassung grenzt Durkheim sich gleich zweifach ab, und zwar gegen Hegels Vorstellung eines allmächtigen Staates und gegen Kants Vorstellung bzw. die des utilitaristischen Individualismus. Dort fände sich genug Material für Debatten über die Hypertrophie des Staates. Aus soziologischer Sicht interessanter ist allerdings die Theorie des Gleichgewichts und der Verschachtelung der Gruppen, die Durkheim anlässlich dieser Analyse der Funktionen des Staates als Garant der individuellen Freiheit gegen den »kollektiven Partikularismus ${ }^{65}$ der Kleingruppen vorlegt. Damit verwirft er die Thesen des liberalen Traditionalismus (und auch die der in einer anderen theoretischen Abstammungslinie stehenden Evolutionstheorie), die die höheren Gruppierungen aus kleinen ursprünglichen Gruppen herleiten, so auch den Staat aus der Familie - was es dieser Tradition der politischen Philosophie erlaubt, gewisse soziale Strukturen als unterdrückerisch und künstlich zu brandmarken. Zugleich verwirft er damit auch die liberale Analyse der Hypertrophie des Staates unter dem zweifachen Einfluss des königlichen Absolutismus und des jakobinischen Zentralismus, die danach streben, die Vielfalt der kleinen und, wie man sagen könnte, halbnatürlichen Gemeinschaften zu eliminieren, die für das Individuum einen Schutzschirm vor der Unterdrückung durch den Staat darstellten. Die zweite Gruppe von Analysen betrifft die Funktion der beschränkten Gruppen für das psychische und moralische Gleichgewicht des Individuums: einerseits als Sozialisationsinstanz, die für die Verinnerlichung sozialer 
Regeln sorgt, andererseits als Ort der Integration und als Identifikationsobjekt. Das sind die beiden Aspekte der Beziehung der Gesellschaft zum Individuum: Repressionsfunktion und Integrations- und Identifikationsfunktion, Sanktion und Fusion. Zweifellos könnte man die anthropologischen Grundlagen der Durkheimschen Soziologie herausarbeiten. Hier wollen wir uns jedoch mit dieser Theorie der Funktion der Sekundärgruppen begnügen (verbunden mit einer Theorie der Krise der Integration in die moderne Gesellschaft, wie sie vor allem in Der Selbstmord erkennbar wird - darauf werden wir noch zurückkommen), einer Theorie, die in der amerikanischen Sozialpsychologie noch zu reichhaltiger Entfaltung kommen wird (Theorie der Kleingruppen) und deren Auswirkungen man heute noch in den aktuellen Diskursen über Geselligkeit und Vereine finden könnte. Durkheims Analyse befragt also die politischen Instanzen nach ihrer Funktion in der Sozialisation der Individuen und gibt der spezifischen Erforschung des politischen Systems und der politischen Institutionen nur wenig Raum.

Die Debatte über die Konstitution des Gegenstandes und die mögliche Präsenz einer vermeintlichen politischen Wissenschaft unter der Maske der Soziologie in Durkheims Werk kann man ohne Bedauern aufgeben. Die Frage nach Durkheims »Politik« ist jedoch mit der Frage nach dem Gegenstand der von ihm angestrebten Wissenschaft noch nicht erschöpft. Diese Frage hat zwei Aspekte. Der eine betrifft seine politischen Interventionen, die praktischen Vorschläge, die er im Namen seiner Soziologie in die politischen Debatten seiner Zeit einbrachte. Der zweite Aspekt betrifft die von ihm vorgeschlagene politische Organisation - die Frage des Korporatismus und des Faschismus und des Verhältnisses zum Sozialismus, die J.-C. Filloux in seinem Buch ausführlich behandelt. Im Blick auf Durkheims politische Praxis, auf seine politischen Diagnosen und Anregungen als Soziologe wissen wir um seine Distanz zur Politik und um die Seltenheit seiner Interventionen. Sie galten dem Eherecht, der Abschaffung bestimmter Regelungen des Erbrechts, dem Streikrecht der Beamten ${ }^{66}$ und der Frage der Gewissensfreiheit im Zusammenhang mit der Dreyfus-Affäre - aber hier ging es eher um Moral als um Politik. ${ }^{67}$ Das wiederkehrende Thema in seinen politischen Ratschlägen betraf Fragen der Berufsgruppen - in Über soziale Arbeitsteilung, explizit und systematisch im zweiten Vorwort von 1902, in Der Selbstmord und in der Physik der Sitten und des Rechts. Man kann darin Vorarbeiten zu einer korporatistischen Doktrin erblicken..$^{68} \mathrm{~J}$.-C. Filloux diskutiert dieses Problem ausführlich, und das zu Recht, denn es ist von zentraler Bedeutung für die Frage nach Durkheims Verhältnis zum Sozialismus. Wir müssen uns hier allerdings vor Anachronismen hüten und dürfen nicht der Logik des Verdachts oder der entgegengesetzten Logik der Apologie verfallen: Erstere könnte von Durkheims Ablehnung des Marxismus (des Klassenkampfes) genährt werden ${ }^{69}$; von seinen Bemühungen, die moralische Autorität des Staates wiederherzustellen und dem religiösen Empfinden eine soziale Grundlage zu geben (was wiederum zu einer Vergottung des Staates führen könnte); letzteres vermöchte auf Durkheims unerschütterlichen Liberalismus zu verweisen, auf seine Billigung der Auflösung der Korporationen ${ }^{70}$, seine Erklärungen zur Legitimation der Trennung zwischen Unternehmerverbänden und Gewerkschaften ${ }^{71}$, seine Sympathien für den Sozialismus, seine Bemühungen um die Bestimmung einer gerechten sozialen Ordnung $^{72}$; und schließlich auch auf die Tatsache, dass Mauss in den Sowjets eine Organisationsform erblickte, die mit Durkheims Ideen zu den berufsständischen Gruppen ebenso vereinbar sei wie mit den Korporationen des Faschismus. Statt sich zum Ankläger oder Verteidiger aufzuwerfen, sollte man die sozialen Vorschläge des 
Soziologen besser zu seiner Theorie in Beziehung setzen und herausarbeiten, inwiefern sie mit seiner Analyse der Krise der modernen Gesellschaft und insbesondere mit der sozialen Frage zusammenhängen. Durkheim weist den berufsständischen Gruppen drei Funktionen zu. Sie haben die Aufgabe, legitime (oder besser: legitimierende) Regulierungs- und Verhandlungsinstanzen für die berufsständischen Beziehungen zu schaffen: eine spezifische Umgebung als ort für Gespräche, in der zwischen Unternehmern und Arbeitnehmern Regeln ausgehandelt und festgelegt werden, die mit Autorität ausgestattet sind und einen »Rechtszustand» in den sozialen Beziehungen herstellen. Sie haben weiter die Aufgabe, ein Sozialisationsmilieu zu schaffen, das zugleich Repressionsinstanz und Identifikationsobjekt ist und einen sanfteren, nähergelegenen Zwang ausübt als Staat oder Gesellschaft, einen Zwang, der stärker ist und über mehr Autorität verfügt als der Zwang, den die häusliche Gruppe in der modernen Gesellschaft auszuüben vermag - ein Objekt zudem, das eher eine Identifikation ermöglicht, weil es näher liegt als die vom Einzelnen allzu weit entfernte Gesellschaft. Und schließlich haben sie die Aufgabe, eine zur Kollektivierung des Eigentums passende Ebene $\mathrm{zu}$ bieten, oberhalb dessen, was einmal das kollektive Familieneigentum war und nun individuelles Privateigentum geworden ist, mit den Ungerechtigkeiten, die das Erbrecht schafft und perpetuiert, aber unterhalb des allzu unpersönlichen kollektiven Staatseigentums, das die Gefahren der Hypertrophie des Staates und der Entwicklung eines parasitären Verwaltungsapparats verstärkt. Wir haben also eine soziale Funktion berufsständischer Regulierung, eine moralische Repressions- und Integrationsfunktion und eine ökonomische Funktion der Kollektivierung des Eigentums. Die den berufsständischen Gruppen zugewiesene Bedeutung erklärt sich aus der Analyse der Entwicklung der häuslichen und der territorialen Bindungen: funktionaler Bedeutungsschwund der häuslichen Gruppe, die auf die Stufe der Kernfamilie reduziert wird; Bedeutungsrückgang der territorialen Bindungen - das Individuum definiert sich weniger durch die Zugehörigkeit zu einer lokalen Gemeinschaft als über die Beziehungen, die es aufgrund seiner Stellung innerhalb einer sozialen Funktionsteilung $\mathrm{zu}$ anderen unterhält. Sie ergibt sich außerdem aus einer Analyse der Besonderheit der modernen Industriegesellschaft (ein von Spencer und Saint-Simon übernommenes Thema): Die ökonomischen Funktionen entwickeln sich auf Kosten der militärischen, administrativen, religiösen und sogar der wissenschaftlichen Funktionen, die zwar wichtig sind, diese Bedeutung aber zu einem Großteil aufgrund ihres Beitrags zur Entwicklung der ökonomischen Funktionen besitzen. Durkheim kann die Idee des Korporatismus bei Schäffle oder vielleicht bei Tönnies gefunden haben. Sie wird von Sorel weiter ausgearbeitet, aber Durkheim ist hier nicht die einzige Quelle. Bei Durkheim haben die Korporationen zwei wesentliche Funktionen: Sie antworten auf das Fehlen oder die Auslöschung der mittleren Gruppen, deren Mittlerfunktion für das Verhältnis zwischen Individuum und Gesellschaft unerlässlich ist. Im ökonomischen Bereich, in dem die Aktivitäten sich anarchisch entwickeln und der unter einem Mangel an Regulierung leidet (Anomie im ersten Sinne des Wortes bei Durkheim, in Über soziale Arbeitsteilung), versprechen sie die Moralisierung des Wirtschaftslebens und die Regulierung der Beziehungen zwischen den Klassen. Das erste Thema antwortet auf die Feststellung einer moralischen Krise, einer Krise der gesellschaftlichen Integration des Individuums („Egoismus« und unkontrollierte Befreiung der Begierden - »Anomie« - oder Verlassenheit); das zweite setzt eine Diagnose hinsichtlich des Wirtschaftslebens als eines Sektors fort, der sich 
anarchisch entwickelt und sich der Kontrolle durch die Gesellschaft entzieht: Krise der Integration der Gesellschaft als Organismus.

Über die Diagnose des Wirtschaftslebens verbindet sich das Thema der berufsständischen Gruppen mit der Analyse des Sozialismus. Durkheim denkt den Sozialismus in zweierlei Weise. In beiden Fällen behandelt er ihn als Symptom der sozialen Entwicklung und der sozialen Krise. Doch im einen Fall sieht er darin den Ausdruck und die Organisation des Klassenkampfes; im anderen dagegen vor allem die Absicht, das Wirtschaftsleben einer Organisation zu unterwerfen. Die sozialistischen Doktrinen können daher entweder in erster Linie als Ausdruck und Versuch einer Lösung der Arbeiterfrage interpretiert werden oder in erster Linie als Formulierung der Forderung nach einer Organisation des Wirtschaftslebens. Als gemeinsame Wurzel für die beiden Interpretationen dient der Begriff der Anarchie; doch das ist nur möglich aufgrund eines Spiels mit den verschiedenen Bedeutungen des Wortes. Was der Soziologe im ersten Aspekt des Sozialismus erkennt, ist das Symptom der anarchischen Begierden in der modernen Industriegesellschaft mangels hinreichend naher und hinreichend starker Regulierungsinstanzen, und hier liegt der Grund für den Rückgriff auf die berufsständischen Gruppen als Sozialisationsmilieus. In diesem Sinne stützt sich die Soziologie auf eine philosophische Anthropologie. ${ }^{73}$ Nach der zweiten analytischen Sicht auf den Sozialismus - die von Durkheim bevorzugt wird (darauf werden wir gleich noch zurückkommen) - bringt der Sozialismus selbst noch in verzerrter oder unvollkommener Form die Notwendigkeit der Organisation eines Sektors des sozialen Lebens zum Ausdruck, nämlich des Wirtschaftslebens, das sich anarchisch und außerhalb der Kontrolle durch das zentrale System oder den Staat entwickelt hat. Hypertrophie, extreme Entwicklung der ökonomischen Funktionen (im Vergleich zu einer Gesellschaft, in der Handel und Industrie noch wenig entwickelt sind), eine vom Staat und den Informations- und Kommunikationsfunktionen losgelöste Entwicklung in dieser Analyse finden sich zahlreiche biologische Metaphern. Das organizistische Schema erlaubt es, das Klassenkampfmodell in den Hintergrund zu rücken. Die Definition der Integration als Integration eines von einem Zentralnervensystem gesteuerten Organismus erlaubt es, die Frage der Integration im sozialen Sinne und die Gefahren, die der »Krieg der Klassen« für diese Integration darstellt, zu neutralisieren oder aufzuschieben. So kommt es, dass Durkheim in seiner Diagnose der sozialen Bewegung, die ihren Ausdruck im Sozialismus findet, nicht den Egalitarismus oder den Kampf gegen die Unterdrückung in den Vordergrund stellt, sondern eher die zentrale Planung als Regulierungserfordernis.

Diese Diskussion über die Stellung politischer Gegenstände in Durkheims Soziologie und über die politische Reichweite dieser Soziologie gibt Anlass, einige Fragen nach dem Projekt $\mathrm{zu}$ stellen, das hinter Durkheims Unternehmen steht und das der soziologischen Tradition möglicherweise weiterhin nachgeht. Um das Verhältnis zur Politik zu klären, reicht es in der Tat nicht aus, wenn man sagt, diese Soziologie schließe die Berücksichtigung politischer Gegenstände aus oder erschwere sie. Ohne Zweifel kann man auf die Ablehnung der politischen Kunst im Namen des wissenschaftlichen Ideals verweisen; auf den Anspruch des Rechts, Umwege zu machen; auf die Ausklammerung unmittelbarer sozialer Probleme im Namen der langfristigen Geduld der Wissenschaft. Man kann auch die These vertreten, die Analyse des Institutionellen und Sozialen in der kristallisierten Form, die sie in Institutionen annehmen, mache es schwierig, soziale Ordnungen als »ausgehandelte« Arrangements zu begreifen, und entwerte die politische Dimension der Phänomene. Schließlich kann 
man auch darauf hinweisen, dass die Definition der sozialen Erfahrung als Zwang, als Widerstand gegen die Bewegungen des individuellen Bewusstseins, die Aufmerksamkeit des Soziologen für die Erforschung jener Mechanismen schwächt, durch die Legitimität zustande kommt oder sich perpetuiert. Das hieße nach den Aspekten zu fragen, die durch die spezifische Sicht des Soziologen auf seine Gegenstände verdunkelt oder an den Rand des Gesichtsfelds gedrängt werden, und unter Zuhilfenahme gewisser Vergleiche und Denkmuster könnte man auch fragen, warum der Durkheimismus - in Comtes Begriffen - die Statik auf Kosten der Dynamik bevorzugt, oder man könnte vergleichen, welche Stellung Durkheim und Weber der politischen Dimension der sozialen Ordnung und der sozialen Arrangements zuweisen. Aber vielleicht sollte man sich nicht mit diesen Vorannahmen begnügen (die Vernachlässigung der Politik) und stattdessen nach den internen Auswirkungen dieser Lücke oder dieser Zensur auf den Gegenstand der Soziologie fragen. Wenn der Gegenstandsbereich der Soziologie ein Bereich des Sozialen ist, der außerhalb des Einflussbereichs der Einigung individueller Willen liegt (um deutlich zu machen, dass die Gesellschaft nicht auf einem Vertrag beruht), wenn der Soziologe vielmehr die Institutionen untersucht, läuft er dann nicht Gefahr, den Konstitutionsprozess zu vernachlässigen? Wenn die Soziologie Phänomene zum Gegenstand hat, die dem individuellen Bewusstsein entgehen oder es übersteigen, "unbewusste« Regulierungen oder Bewegungen, wird es dem Soziologen da nicht schwerfallen, den Debatten über die sozialen Subjekte ihre Konsistenz und Reichweite wiederzugeben? Und vor allem schließlich, wenn der Soziologe einen Bereich des Sozialen abgrenzt, in dem er unterhalb der atomisierten politischen Subjekte soziale Ensembles zu finden vermag, riskiert er dann nicht, in eine Topik (»Ebenen« der sozialen Realität) zu verwandeln, was eigentlich eine provisorische Karte des Wissens und der Forschungsgebiete ist, hier definiert im Rahmen eines expliziten und impliziten wissenschaftlichen Streits mit dem traditionsverhafteten Denken? Und riskiert er damit nicht, aus dem von ihm untersuchten Bereich der Regulierungen Phänomene des politischen Kampfes auszuschließen, die er dann als Strategien nur mit Mühe wieder einführen könnte?

Man wird bemerkt haben, dass man diese Fragen nach der Wahl des Gegenstandes auch im Blick auf das Verhältnis zwischen der Soziologie und anderen Fachgebieten formulieren könnte: das Verhältnis zum Recht und zur Philosophie in erster, zur Psychologie in zweiter, zur Geschichtsphilosophie und den »Wissenschaften der Moral und der Politik « in dritter Linie. Auch hier wieder ist der Hinweis angebracht, dass wissenschaftliche Gegenstände nicht in der Einsamkeit eines intellektuellen Gründungsentwurfs konstruiert werden. Die Frage nach der Stellung des politischen objektbereichs bei Durkheim ist daher belastet vom Substantialismus (Setzung eines politischen »Objekts«), ganz zu schweigen vom Chorgeist oder vom Geist des akademischen Berufsstandes. Sie ist jedoch interessant, wenn sie die Möglichkeit eröffnet, die Durkheimsche Soziologie nach ihrer Blindheit für die politischen Kämpfe zu befragen. Darum ging es in den oben formulierten Fragen. Aber nachdem der Soziologe vor der eigenen Haustür gekehrt hat, darf er seine akademischen Nachbarn bitten, nun auch einen Blick vor ihre Haustür zu werfen und nachzusehen, welche Scholien die ihre versperren. Hat die Zensur der Politik in der Durkheimschen Soziologie nicht dazu geführt, dass man andernorts eine "Wissenschaft» schwatzen lässt, die für diese »Kunst« der Politik geschaffen worden ist - um noch einmal die von Mauss benutzten Ausdrücke aufzugreifen? 


\section{Soziale Frage und jüdische Frage}

40 Das obige Exposé hat einige der großen Gegensätze vorgestellt, um die herum Durkheims Soziologie aufgebaut ist, ohne darauf einzugehen, wie sie sich in der Zeit organisiert. Darauf müssen wir nun etwas systematischer zu sprechen kommen - was wiederum heißt, dass wir die Frage nach der Wende oder den Wenden in seinem Werk wiederaufgreifen. Um die Diskussion zu zentrieren, werden wir sie auf die Zeit von 1885 (Durkheims ersten Rezensionen) und 1898 (dem Erscheinungsdatum des ersten Bandes der Année sociologique, der auf Über soziale Arbeitsteilung, Regeln der soziologischen Methode und Der Selbstmord folgte) einschränken und die These vertreten, dass Durkheim von einer soziologischen, an der sozialen Frage orientierten Fragestellung zu einer Definition der Krise der modernen Gesellschaft als moralischer Krise übergeht; dass in der Diagnose dieser moralischen Krise der Klassenantagonismus zunehmend dem Wertewiderspruch innerhalb der Bourgeoisie weicht; und dass schließlich in die Analyse dieses Widerspruchs auf weitgehend implizite Weise eine Reflexion über die jüdische Frage im Frankreich seiner Zeit Eingang findet.

41 Die soziale Frage steht in Durkheims ersten Werken, insbesondere dem Buch Über soziale Arbeitsteilung, im Zentrum der Fragestellung. Diese Frage umfasst, wie wir gesehen haben, zwei Fragen: einerseits die nach dem sozialen Band, den Grundlagen einer sozialen Ordnung, und andererseits die Arbeiterfrage. Während die erste weiterhin im Zentrum seiner Fragestellung bleibt, wird die zweite langsam verdrängt oder verschoben. In Über soziale Arbeitsteilung (dessen Lektüre sich meist auf die beiden ersten Bücher beschränkt, während das dritte Buch »Über die anormalen Formen" weniger Beachtung findet) befasst Durkheim sich ausführlich mit dem Krieg der Klassen. In Le Socialisme gibt Durkheim der "sozialen« Frage (in der ersten der beiden oben skizzierten Bedeutungen) den Vorzug gegenüber der »Arbeiterfrage«. Bei letzterer handelt es sich in seinen Augen in erster Linie um eine moralische Frage. Es geht darum, dass die effektive Organisation der Entlohnungen gerecht ist und, vor allem, dass die Mechanismen zur Erörterung, Einführung und Verinnerlichung dieser Verteilungsregeln so wirkungsvoll sind, dass »die Menschen sich mit ihrem Los abfinden.$^{74}$ Das ist der Sinn der Analysen und der Empfehlungen bezüglich der berufsständischen Gruppen. So gesehen, ist die Arbeiterfrage in erster Linie ein Problem der Regulierung (oder Unterdrückung) von Ansprüchen.

Diese Serie von Verschiebungen erhält ihre Bedeutung natürlich vor dem Hintergrund der Geschichte des Sozialismus (als Theorie wie als politische und soziale Bewegung) in Frankreich. Sie erfolgen im Zuge des intellektuellen Weges, der von der Arbeitsteilung (1893) über die Vorlesungen zum Sozialismus (1895) bis hin zum zweiten Vorwort der Arbeitsteilung führt.

Aber es gibt mehr als eine Bewegung in Durkheims Fragestellung und Interessen. Die Verschiebung der sozialen Frage oder, wie Durkheim sagt, der »Arbeiterfrage« hin zur moralischen Frage wird mit der Analyse des Selbstmords von einer empirischen Vertiefung und Spezialisierung in der statistischen Erforschung der Moral begleitet. Man hat es sich angewöhnt (man erinnere sich an meine Bemerkungen über die strenge Einteilung der »Genres« in der Literatur und mehr noch in der soziologischen Kritik), darin eine Übung in empirischer Forschung, ein methodologisches Modell, zu erblicken. Doch als Untersuchung eines Symptoms der moralischen Krise und als Analyse der sozialen Formen der Regulierung von Ansprüchen und von Formen des 
Verhältnisses zwischen Individuum und Gruppe steht dieses Buch in tiefer Kontinuität zu Durkheims Forschungen hinsichtlich der sozialen und moralischen Krise der Industriegesellschaften. Die Analyse der Ursachen des Selbstmords enthält tatsächlich - symmetrisch zur Erforschung der mangelhaften (übermäßigen oder unzureichenden) Integration und der Mängel der Repression - eine Untersuchung der Formen eines guten Lebens in der modernen Gesellschaft. Diese Untersuchung erfolgt in Gestalt einer Reflexion über die Werte, die in verschiedenen berufsständischen Gruppen herrschen sollten. In dieser Reflexion, in der man leicht Übereinstimmungen mit Webers Gedanken zum Ethos von Klassen oder Gruppen oder mit Webers und - darüber hinaus - Nietzsches Philosophie hinsichtlich der Unvereinbarkeit verschiedener Ethiken entdecken könnte, finden zwei Arten von Verschiebungen gegenüber Durkheims ursprünglichen Überlegungen zur sozialen Frage ihren Ausdruck. Zunächst ein Übergang von Überlegungen bezüglich des Klassenkampfs zu einer Reflexion über die Werte der verschiedenen Gruppen innerhalb der Bourgeoisie (Geschäftsleute, Militärs, Intellektuelle usw.). Sodann - innerhalb dieser Reflexion und sie überdeterminierend eine Meditation über die Möglichkeit, ein gut assimilierter Jude im Frankreich der Zeit zu sein - eine Frage, die zweifellos durch die Anfänge der Dreyfus-Affäre zugespitzt und vertieft wurde. Jemandem, der das Buch über den Selbstmord nur in Readern oder vermittelt über methodologische Kommentare liest, mag das gewagt erscheinen. Doch die bloße Lektüre der Texte und deren zeitliche Einordnung veranlassen dazu, sich nicht mit den klassischen Darstellungen zu begnügen, die Durkheims Reaktion auf die Dreyfus-Affäre dem sorgfältig markierten und abgegrenzten Bereich der politischmoralischen Stellungnahmen zuordnen (im Augenblick der Unterzeichnung der Petition), wodurch das Werk den Fragen, die der jüdische Hochschullehrer sich stellt, fremd bleibt. Wir glauben dagegen, man kann durchaus fragen (und der Vergleich der Analysen, die Durkheim 1888 in einer ersten Arbeit über den Selbstmord gibt, mit denen des Buchs von 1897 berechtigt zu dieser Frage), ob Der Selbstmord nicht auch eine von der Dreyfus-Affäre ausgelöste und dramatisierte Reflexion über den Unterschied der ethischen Einstellungen ist, die von einem Soldaten und von einem Hochschullehrer erwartet werden, wodurch klar wird, dass Dreyfus kein Feigling ist, wenn er sich anklagen lässt; und ob es sich nicht allgemeiner noch um eine heimlich seit langem verfolgte Reflexion über die Integration der Juden in das Frankreich der Zeit handelt. Durch eine Analyse der in Der Selbstmord gewählten Beispiele (Vorhandenes und Fehlendes, das eine Zensur verrät) könnte man zeigen, dass dies einer der Augenblicke der Rückkehr der jüdischen Kultur und Tradition in Durkheims Bewusstsein ist. Aber das ist ein anderes Rätsel ...

\section{BIBLIOGRAPHIE}

Alpert, H. (1939): Emile Durkheim and his Sociology, Columbia University Press: New York.

Aron, R. (1967): Les Etapes de la pensée sociologique, Gallimard: Paris.

Ben-David, J. (1971): The Scientist's Role in Society, Englewood Cliffs, N.J.: Prentice-Hall. 
Bénichou, P. (1973): Le Sacre de l'écrivain, 1750-1830. Essai sur l'avènement d'un pouvoir spirituel laïque dans la France moderne, Paris: J. Corti.

Besnard, P. (1973): »Durkheim et les femmes ou le Suicide inachevé«, Revue française de sociologie, 14 (1), S. 27-61.

Birnbaum, P. (1976): „La conception durkheimienne de l'Etat: l'apolitisme des fonctionnaires«, Revue française de sociologie, 17, S. 247-258.

Boudon, R. / Bourricaud, F. (1983): Dictionnaire critique de la sociologie, Paris: PUF.

Bouglé, C. (1899): Les Idées égalitaires, étude sociologique, Paris: F. Alcan.

Bouglé, C. (1908a): Essais sur le régime des castes, Paris: F. Alcan.

Bouglé, C. (1908b): »Marxisme et sociologie«, Revue de métaphysique et de morale, XVI (6), S. 723-

750 .

Boutroux, E. (1874): De la contingence des lois de la nature, Paris: Germer-Baillière.

Cassagne, A. (1906): La Théorie de l'art pour l'art en France chez les derniers romantiques et les premiers réalistes, Paris: Hachette.

Chamboredon, J.-C. (1983): „Le temps de la biographie et les temps de l'histoire. Remarques sur la périodisation; à propos de deux études de cas«, in: Fritsch, P. (Hg.): Le Sens de l'ordinaire, Paris: C.N.R.S., S. 17-29 und $212 \mathrm{f}$.

Clark, T. (1973): Prophets and Patrons, The French University and the Emergence of the social Science, Cambridge/Mass.: Harvard University Press.

Cuvillier, A. (1953): Où va la sociologie française? Avec une étude d'Emile Durkheim sur la sociologie formaliste, Paris: Libr. M. Riviere.

Cuvillier, A. (1962): Manuel de sociologie, Paris: PUF.

Davy, G. / Moret, A. (1924): Eléments de sociologie, Bd. I: Sociologie politique, Paris: Delagrave.

Davy, G. / Moret, A. (1923): Des clans aux Empires, Paris: La Renaissance du livre.

Davy, G. (1973): L’Homme, le fait social et le fait politique, Paris / Den Hag: Mouton.

Davy, G. (1950 [1931]): Sociologues d'hier et d'aujourd'hui, 2. veränderte und erweiterte Aufl., Paris: PUF.

Digeon, C. (1959): La Crise allemande de la pensée française (1870-1914), Paris: PUF.

Durkheim, E. (1898): »Préface«, Année sociologique, I, S. I-VII.

Durkheim, E. (1923): Le Conflit de la morale et de la sociologie, 3. Aufl., Paris: Alcan.

Durkheim, E. (1928): Le Socialisme: sa définition, ses débuts, la doctrine saint-simonienne, hg. und mit einem Vorwort von M. Mauss, Paris: F. Alcan.

Durkheim, E. (1970): La Science sociale et l'action, hg. von J.-C. Filloux, Paris: PUF.

Durkheim, E. (1970 [1885a]): »Les études de science sociale«, in: Ders. (1970), S. 184-214.

Durkheim, E. (1970 [1885b]): Rezension des Buches von A. Fouillée, La Propriété sociale et la démocratie, in: Durkheim (1970), S. 171-183.

Durkheim, E. (1970 [1887]): »La conception matérialiste de l'histoire«, in: Ders. (1970), S. 246-255.

Durkheim, E. (1970 [1893]): »Note sur la définition du socialisme«, in: Ders. (1970), S. 227-236.

Durkheim, E. (1970 [1900]): »La sociologie en France au XIX ${ }^{e}$ siècle«, in: Ders. (1970), S. 111-136. 
Durkheim, E. (1970 [1909]): »Sociologie et sciences sociales«, in: Ders. (1970), S. 137-159.

Durkheim, E. (1975): Textes, 3 Bde., hg. von V. Karady, Paris: Eds. de Minuit.

Durkheim, E. (1975 [1895]): »Lo stato attuale degli studi sociologici in Francia«, in: Ders. (1975), Bd. 1, S. 73-108.

Durkheim, E. (1975 [1899]): »Une révision de l'idée socialiste«, in: Ders. (1975), Bd. 3, S. 163-172.

Durkheim, E. (1975 [1908a]): »Débat sur le rapport entre les fonctionnaires et l'état «, in: Ders.

(1975), Bd. 3, S. 189-202.

Durkheim, E. (1975 [1908b]): »Débat sur les syndicats de fonctionnaires«, in: Ders. (1975), Bd. 3, S. 202-217.

Durkheim, E. (1975 [1915]): »La sociologie«, in: Ders. (1975), Bd. 1, S. 109-118.

Durkheim, E. (1975 [1900]): »La sociologia e il suo dominio scientifico«, in: Ders. (1975), Bd. 1, S. 13-36.

Durkheim, E. (1975 [1920]): »Introduction à la morale«, in: Ders. (1975), Bd. 2, S. 313-331.

Durkheim, E. (1983 [1897]): Der Selbstmord, übers. von S. und H. Herkommer, Frankfurt/M.:

Suhrkamp.

Durkheim, E. (1986 [1898]): »Der Individualismus und die Intellektuellen«, in: Bertram, H. (Hg.): Gesellschaftlicher Zwang und moralische Autonomie, Frankfurt/M.: Suhrkamp, S. 54-70.

Durkheim, E. (1988 [1930]): Über soziale Arbeitsteilung. Studie über die Organisation höherer Gesellschaften, übers. von L. Schmidts, durchges. von M. Schmid, Frankfurt/M.: Suhrkamp.

Durkheim, E. (1991 [1950]): Physik der Sitten und des Rechts. Vorlesungen zur Soziologie der Moral, übers. von. M. Bischoff, Frankfurt/M: Suhrkamp.

Durkheim, E. / Fauconnet, P. (1975 [1903]): „Sociologie et sciences sociales«, in: Ders. (1975), Bd. 1, S.121-159.

Duvignaud, G. (1973): L'Anomie, hérésie et subversion, Paris: Anthropos.

Fabiani, J.-L. (1980): La Crise du champ philosophique (1880-1914). Contribution à l'histoire sociale du système d'enseignement, Paris: Laboratoire de sciences sociales E.N.S.

Feyerabend, P. (1976): Wider den Methodenzwang. Skizze einer anarchistischen Erkenntnistheorie, übers. von H. Vetter, Frankfurt/M.: Suhrkamp.

Filloux, J.-C. (1977): Durkheim et le socialisme, Paris / Genève: Ed. Droz.

Gaudemar, P. de (1969): »E. Durkheim, sociologue de l'éducation«, Homo (Annales de la Faculte de Toulouse), VIII, Okt., S. 129-142.

Goody, J. (1979): La raison graphique, Paris: Eds. de Minuit.

Isambert, F.-A. (1973): »Durkheim et la statistique écologique », in: Une nouvelle civilisation, Hommage à Georges Friedmann, Paris: Gallimard, S. 93-110.

Karady, V. (1972): »Normaliens et autres enseignants de la Belle Epoque: note sur l'origine sociale et la réussite dans une profession intellectuelle«, Revue française de sociologie, 13 (1), S. 35-48.

Lacroix, B. (1981): Durkheim et le politique, Paris: Presses de la Fondation nationale des sciences politiques.

Lavisse, E. (1882): » L'enseignement historique en Sorbonne et l'éducation nationale«, Revue des Deux Mondes, XLIX, S. 870-897. 
Lindenberg, D. (1975): Le marxisme introuvable, Paris: Calmann-Lévy.

Lukes, S. (1972): Emile Durkheim, his Life and Work. A Historical and Critical Study, New York / London: Rarper and Row.

Mauss, M. (1924): »Appréciation sociologique du bolchévisme«, Revue de métaphysique et de morale, 31, S. 103-132.

Mauss, M. (1925): „Socialisme et bolchévisme«, Le monde slave, Neue Serie 2, Februar, S. 201-222.

Mauss, M. (1968): Euvres, Bd. 1, hg. von V. Karady, Paris: Eds. de Minuit.

Mauss, M. (1969): CEuvres, Bde. 2 und 3, hg. von V. Karady, Paris: Eds. de Minuit.

Mauss, M. (1969 [1920]): »La Nation«, in : Ders. (1969), Bd. 3, S. 573-625.

Mauss, M. (1969 [1925]): »L'œuvre inédite de Durkheim et de ses collaborateurs«, in: Ders. (1969), Bd. 3, S. 473-499.

Mauss, M. (1969 [1927]): »Divisions et proportions des divisions de la sociologie«, In: Ders. (1969), S. $178-245$.

Mauss, M. / Fauconnet, P. (1969 [1901]): »Sociologie«, in : Ders. (1969), Bd. 3, S. 139-177.

Mauss, M. (1928): Vorwort zu Durkheim (1928).

Mayeur, J.-M. (1973): Les Débuts de la Troisième République, 1871-1898, Paris: Seuil.

Merton, R. K. (1995 [1939]): „Soziale Struktur, Anomie und Devianz«, in: Ders.: Soziologische Theorie und soziale Struktur, übers. von H. Beister, Berlin / New York: de Gruyter.

Nandan, Y. (1974): Le maître, les doctrines, les membres et le magnum opus, une étude critique et analytique de l'école durkheimienne et de l'Année sociologique, thèse de $3^{\mathrm{e}}$ cycle, Sorbonne.

Nisbet, R. A. (1974): The Sociology of Emile Durkheim, New York: Oxford University Press.

Parsons, T. (1937): The Structure of Social Action, New York: McGraw-Hill.

Ranulf, S. (1938): „Scholarly forerunners of fascism«, Ethics, 50, S. 16-34.

Selvin, H. (1966): »Aspects méthodologiques du Suicide«, in: Boudon, R. / Lazarsfeld, P. (Hg.): Méthodes de la sociologie, Bd. 2: L'analyse empirique de la causalité, Paris / Den Hag: Mouton, S. 276291.

Vogt, P. (1976): The Politics of Academic Sociological Theory in France, 1890-1914, Xerox University Microfilms, Ann Arbor, Michigan.

Wolff, K. (Hg.) (1960): Emile Durkheim, 1858-1917. A Collection of Essays, With Translations and a Bibliography, Columbus: The Ohio State University Press.

\section{NOTES}

1. Cuvillier (1953) sowie Davy (1950) und (1972) zeugen davon noch in den beiden letzten Jahrzehnten.

2. Parsons (1937), Alpert (1939) und schließlich Lukes (1973) in einer werkorientierten Biographie.

3. Insbesondere Clark (1973) und diverse Sondernummern der Revue française de sociologie (A propos des Durkheim, 1976 ; Les durkheimiens, 1979 ; Sociologies françaises au tournant du siècle, 1981).

4. Z. B. Besnard (1973), Gaudemar (1969), Isambert (1973)

5. Z. B. Selvin (1966). 
6. Parsons (1937).

7. Nisbet (1975). Diese angelsächsischen Interpretationen sind von einer jüngeren analytischen Linie zu unterscheiden, die aus dem Anomiebegriff eine Begeisterung für die unter der sozialen "Ordnung« liegende »Unordnung« bzw. "Chaos« ableitet. Dazu gehören Post-Gurvitchianer wie Duvignaud (1973) und Balandier, die das von Parsons und Merton entwickelte strukturfunktionalistische Bild Durkheims erschüttern.

8. Ben-David (1971); Clark (1973).

9. Dies gilt für die Arbeiten Karadys ([1972], sowie seine Beiträge in den erwähnten Sondernummern der Revue française de sociologie) wie für die von Vogt (1976). Den allgemeinen Rahmen dieser Soziologie der wissenschaftlichen Disziplinen liefert Ben-David (1971).

10. Lindenberg (1975).

11. Aron (1967).

12. Filloux (1977); Lacroix (1981).

13. Der Ausdruck »Sozialwissenschaft«, so wie er zum Zeitpunkt, da Durkheim zu arbeiten beginnt, gewöhnlich verwendet wird (etwa in Titeln von Werken von Coste oder Fouillé, zu denen Durkheim seine ersten Rezensionen schreibt), bezeichnet die philosophische Behandlung eines Komplexes sozialer Fragen. In Bordeaux hielt Durkheim seine erste Vorlesung in "Sozialwissenschaft« (siehe Durkheim [1975], S. 73 f.)

14. Durkheim (1970 [1900]), S. 136.

15. Durkheim (1970 [1900]), S. $111 \mathrm{ff}$.

16. Durkheim (1975 [1895]), Bd. I, S. 105.

17. Durkheim (1970 [1909]), S. 141. Die gleichen Gründe erklären auch Durkheims Misstrauen gegenüber biografischen Methoden: sie stehen im Verdacht, an den Illusionen der "historisierenden« Geschichte teilzuhaben bzw. solche zu nähren.

18. Dies trifft nicht zuletzt auf Clark (1973) zu; aber er ist nicht der einzige.

19. Vgl. Fabiani (1980).

20. Boutroux (1874), Philosophieprofessor Durkheims an der Ecole normale supérieure. Und nach Davy (1973, S. 17-23) zusammen mit Fustel de Coulanges einer der wenigen, die ihn dauerhaft geprägt haben.

21. Goody (1979).

22. Vgl. Feyerabend (1976).

23. Durkheim (1923), S. $361 \mathrm{f}$.

24. Durkheim (1975), Bd. II, S. 455.

25. Mauss (1928), S. 7 f.

26. Zum Verhältnis der sich akademisch etablierenden Soziologie zum Marxismus in der 1890er Jahren siehe das interessante Zeugnis von Bouglé (1908b).

27. Durkheim (1928), S. 348.

28. Durkheim (1928), S. 351.

29. Dies ist ein neuer, unerwarteter und paradoxer Verdienst der Psychoanalyse: sie ermöglicht es, sich der (für den kritischen Diskurs und die Exegese) »nutzlosen« und hemmenden Zensur durch die Ideengeschichte zu entledigen.

30. Siehe Bouglé (1899), Bouglé (1908), Mauss (1969 [1920]), Mauss (1924), Mauss (1925), Davy (1924) und Davy / Moret (1923).

31. Zum Verhältnis von Einzel- und sektorialer Geschichte anhand eines konkreten Falls vgl. Chamboredon (1983).

32. Vgl. Bénichou (1973); vgl. auch Cassagne (1906) in Bezug auf den Mythos des Mandarinentums in der Philosophie der Schule des l'Art pour l'art.

33. Zur Säuberung in der Verwaltung nach der Krise vom 16. Mai 1877 siehe Mayeur (1973).

34. Zur intellektuellen Rolle Deutschlands in Frankreich zwischen 1870 und 1914 siehe Digeon (1959). 
35. Vgl. Durkheim (1970 [1885b]).

36. Durkheim 1991 [1950]): Durkheim hielt die Vorlesungen zwischen 1890 und 1900 in Bordeaux, dann 1904 und 1912 an der Pariser Sorbonne.

37. Durkheim (1975 [1920]); Mauss' Einleitung S. 313 f.

38. Durkheim (1991 [1950]), S. 12 ff.

39. Durkheim (1970 [1885a]).

40. Durkheim (1975 [1895]).

41. Durkheim (1898), S. I-IV.

42. Durkheim (1970 [1900]).

43. Durkheim (1975 [1900]).

44. Mauss / Fauconnet (1969 [1901]).

45. Durkheim / Fauconnet (1975 [1903]).

46. Durkheim (1970 [1909]).

47. Durkheim (1975 [1915]).

48. Durkheim (1970 [1885a]), S. 197.

49. Durkheim (1970 [1885a]), S. 203.

50. Durkheim (1970 [1885a]), S. 214.

51. Durkheim (1970 [1885a]), S. 203.

52. Durkheim (1975 [1895]), S. 91.

53. Die tatsächliche Praxis (abgedeckte Themenfelder, behandelte Themen) könnte anhand der Liste und einer Klassifikation der rezensierten Titel analysiert werden. Das Instrument, die Kompilation des Inhaltsverzeichnisses der Année sociologique, gibt es: Nandan (1974).

54. Durkheim (1898), S. I.

55. Durkheim (1898), S. II f.

56. Durkheim (1898), S. IV.

57. Vgl. Mauss (1969 [1927]).

58. Vgl. Mauss (1969 [1925]).

59. Mauss (1969 [1927]), S. 185 ff.

60. Mauss (1969 [1927]), S. 235.

61. Mauss (1969 [1927]), S. 237.

62. Durkheim (1991 [1950]), S. 75.

63. Vgl. Birnbaum (1976), S. 254.

64. Vgl. Bouglé (1899), Bouglé (1908a), Mauss (1924), Mauss (1925), Mauss (1969 [1920]), Davy / Moret (1924), Cuvillier (1962).

65. Durkheim (1991 [1950]), S. 92.

66. Durkheim (1975 [1908a]) und Durkheim (1975 [1908b]).

67. Durkheim (1986 [1898]).

68. Vgl. Ranulf (1938). Vgl. auch den Briefwechsel zwischen Mauss und Ranulf in Études durkheimiennes, Februar 1983, Nr. 8, S. 1-4.

69. Zu Durkheims Beziehung zum Marxismus vgl. Durkheim (1970 [1897]), Mauss (1928), Durkheim (1970 [1893]) sowie Durkheim (1975 [1899]).

70. Durkheim (1988 [1930]), S. 46.

71. Durkheim (1988 [1930]), S. 47, 67 (Anm. 34). Siehe auch die Verurteilung einer Abhängigkeit der Korporation vom Staat, S. 49.

72. Durkheim (1988 [1930]), S. 73.

73. Sie wird vor allem in Durkheims Der Selbstmord (1988 [1897]) entwickelt.

74. Durkheim (1928), S. 291. 
INDEX

Mots-clés : le social, morale, politique, Emile Durkheim

Schlüsselwörter : das Soziale, Moral, Politik, Emile Durkheim

\section{AUTEURS}

\section{JEAN-CLAUDE CHAMBOREDON}

Jean-Claude Chamboredon (geb. 1930), Soziologe. Nähere Informationen finden Sie hier. 